\title{
Chronotypes ou géotypes? Discussion sur quelques outils néolithiques peu fréquents du nord-ouest de la France
}

Chronotype or geotype? A discussion on rare Neolithic tools in the north-west of France

\section{Dominique Prost}

\section{OpenEdition Journals}

Édition électronique

URL : http://journals.openedition.org/rao/5837

DOI : 10.4000/rao.5837

ISSN : 1775-3732

Éditeur

Presses universitaires de Rennes

\section{Édition imprimée}

Date de publication : 18 décembre 2020

Pagination : 23-43

ISBN : 978-2-7535-8232-3

ISSN : 0767-709X

Référence électronique

Dominique Prost, «Chronotypes ou géotypes ? Discussion sur quelques outils néolithiques peu fréquents du nord-ouest de la France », Revue archéologique de l'Ouest [En ligne], 36 | 2020, mis en ligne le 18 décembre 2020, consulté le 02 avril 2021. URL : http://journals.openedition.org/rao/5837 ; DOI : https://doi.org/10.4000/rao.5837 


\title{
Chronotypes ou géotypes? \\ Discussion sur quelques outils néolithiques peu fréquents du nord-ouest de la France
}

\author{
Chronotype or geotype? A discussion on rare Neolithic tools in the north-west of France
}

\author{
Dominique Prost ${ }^{a}$
}

\begin{abstract}
Résumé : Des outils rares, en marge du fonds commun de l'outillage néolithique du nord-ouest de la France, ont été mis en évidence depuis quelques années, voire plusieurs décennies, mais sont restés en grande partie inconnus de la communauté scientifique. Provenant essentiellement de sites normands, ils sont proposés ici pour tenter d'être reconnus comme de nouveaux morphotypes qui viendraient compléter la liste typologique en vigueur. Ces outils qui ont été nommés provisoirement becs biseautés, burins transversaux, couteaux demi-lune, couteaux quadrangulaires, pointes laminaires à coches basales et herminettes à encoches, pourraient enrichir la discussion entre lithiciens pour savoir s'ils peuvent aussi servir par la suite de chronotypes (c'est-à-dire en vue d'être utilisés comme marqueurs chronoculturels) et/ou de géotypes (c'est-à-dire en vue d'être utilisés comme marqueurs d'un territoire donné).
\end{abstract}

\begin{abstract}
Rare tools not within the common range of Neolithic tools in north-west France, have been documented for several years, or even decades, but still remain largely ignored by the scientific community. These tools are mainly found on sites in Normandy, which suggests that they can be considered as new morphological types to be added to the current typological list. These tools are temporarily called bevelled becs, transverse burins, $1 / 2$ moon knives, quadrangular knives, blade points with basal notches, and notched adzes. Further discussion amongst lithics specialists is needed in order to establish whether they can be further considered as chronocultural markers (chronotypes) or as territorial markers (geotypes).
\end{abstract}

Mots clés : Néolithique, outils marginaux, typologie, morphotypes, chronotypes, géotypes, Normandie, nord-ouest de la France.

Keywords: Neolithic, marginal tools, typology, formtypes, chronotypes, geotypes, Normandy, north-west of France.

\section{Présentation}

Depuis plusieurs années, voire des décennies, certains outils par leur aspect marginal, leur quantité rare par site, ont pu échapper à l'attention des néolithiciens ou les laisser interrogatifs, ne leur trouvant aucune place distincte à leur accorder dans les listes typologiques conventionnelles. Nous avons retenu dans le cadre de cette publication six d'entre eux dont la forme, les dimensions, éventuellement leur fonction, pourraient faire d'eux des morphotypes à part, donc d'être susceptibles de pouvoir les identifier potentiellement comme tels dans l'étude typologique des outils du nord-ouest de la France par le fait de leur présence sur plusieurs sites. La majorité d'entre eux provient de sites normands mais aussi de celui du Néolithique final de Bettencourt-Saint-Ouen dans la Somme et plus rarement de Seine-et-Marne. Nous n'avons aucune prétention à couvrir un secteur géographique précis de façon exhaustive. La détermination de ces outils dépend essentiellement des découvertes fortuites faites au cours des pérégrinations de nos activités d'archéologue en archéologie préventive, à l'Afan puis à l'Inrap, depuis les années 1980. C'est, au gré du hasard, leur répétition sur diverses occupations néolithiques qui nous a fait prendre peu à peu conscience de les distinguer autrement que des outils dits indéterminés ou retouchés.

a Archéologue Inrap, Inrap Normandie (dominique.prost@inrap.fr) 
Notre objectif est donc de les faire connaître à la communauté des néolithiciens. Ceux de Normandie les connaissent en partie. Mais leur reconnaissance comme morphotype à part demanderait à être validée (ou non) par d'autres néolithiciens. C'est bien l'objectif que nous nous donnons ici. Nous discuterons ensuite quelle pourrait être la valeur chronotypologique qu'on pourrait leur attribuer et quelle démarche à suivre pour y parvenir. La recherche de leur extension géographique fait partie également de nos préoccupations pour l'avenir de ces outils qui pourraient, au terme d'une étude plus approfondie, fournir des informations d'ordre géotypologique. À l'heure ou certaines cultures sont remises actuellement en question ou encore mal identifiées, nous sommes toujours à la recherche de nouveaux apports d'indices chronoculturels pour les étudier. Un enrichissement de la liste typologique de l'outillage lithique pourrait y contribuer.

\section{NOUVEAUX APPORTS MORPHOTYPOLOGIQUES}

\section{Les becs biseautés}

Les pièces à bec biseauté - qui ne portaient pas encore ce nom lors de leur découverte - ont été mises au jour pour la première fois à Marne-la-Vallée (Seine-et-Marne, projet d'extension du parc Disneyland) en 1992 sur une des nombreuses concentrations de mobilier trouvées en prospection de surface dont nous avions la charge de faire l'étude (Prost, 1992). Six pièces dont quatre en provenance de Coupvray "La Mézière-Sud » (id., p. 301) et deux de Coupvray "Les Regards"(id., p. 292), sur la même commune, avaient retenu notre attention. Faute de référence bibliographique, nous les avions enregistrées comme pièces typologiquement indéterminées tout en précisant "pièces à bord tranchant étroit dégagé ». Par la suite, au cours de l'étude de l'outillage du site néolithique de Bettencourt-Saint-Ouen (Somme), nous avons identifié une série d'outils peu communs intitulés de manière semblable "pièces à tranchant dégagé " pour lesquelles nous remarquions des similarités avec celles de Marne-la-Vallée (Prost in Martin, 1996, p. 62). Au nombre de 41 pièces, ce lot regroupait tout à la fois un mélange de pièces à museau, à languette, à épaulement, traduisant une variabilité dans la délinéation de ces outils dont la distinction typologique restait encore très floue pour nous. On a pu constater toutefois un point commun. C'étaient des outils que l'on trouvait presque exclusivement en surface, se répartissant de façon diffuse sur le sol comme les grattoirs dans les zones de fortes concentrations là où devait se développer la plus grande partie des activités domestiques. Il a fallu attendre la fouille du site chasséen de Bernières-sur-Seine
"Les Fondriaux » (Eure) pour identifier avec plus de précision ce type d'outils en établissant d'étroites similitudes avec celles de Marne-la-Vallée et certaines pièces de BettencourtSaint-Ouen, suggérant l'existence d'un morphotype bien distinct. On pourra toutefois s'interroger avec raison pour savoir si certains d'entre eux ne seraient pas des perçoirs dont la pointe aurait été cassée, ce qui n'est pas exclu. C’est pourquoi une étude typologique détaillée associée à une analyse tracéologique à faible grossissement a permis d'en préciser définitivement les caractéristiques morphotypologiques. C'est à partir de ce moment-là que nous avons choisi de leur attribuer le nom de "bec biseauté ". Comme nous avons eu l'occasion de le dire récemment, il s'agit d'un terme provisoire dans l'attente de leur donner un nom définitif qui puisse faire l'unanimité (Prost et al., 2017, p. 363) ${ }^{1}$.

Ces outils ont tous en commun, comme support, des éclats relativement grands (de 6 à $9 \mathrm{~cm}$ de long), le plus souvent entiers, conservant fréquemment des plages corticales résiduelles. Ils se caractérisent par une saillie distale ou latéro-distale en forme de bec, dégagée par retouches directes abruptes, voire par encoche (fig. 1). Le bec se termine par un biseau épais qui comporte une retouche fine et/ou des traces d'utilisation, ce qui écarte l'hypothèse d'un perçoir cassé. Ce bec est plus ou moins saillant selon les cas, entre 1 à $3 \mathrm{~cm}$ de long et 0,7 à $1,5 \mathrm{~cm}$ de large, en général. On constate que ces becs sont souvent en position dextre (fig. $1, \mathrm{n}^{\text {os }} 1$, 2 4, 5, 7 et 8). Le bord latéral gauche qui s'oppose au bec est souvent constitué d'un dos partiel retouché ou cortical qui semble avoir été façonné ou recherché pour faciliter la prise en main (fig. 1, $\mathrm{n}^{\text {os }} 1,5,7,8$ ). Sur le plan fonctionnel, ces outils auraient agi sur la matière d'œuvre par percussion posée transversale, par raclage ou grattage. L'étroite largeur du biseau leur confère l'avantage de pouvoir amplifier la force mécanique de pénétration, leur épaisseur leur assurant plus de solidité. Si les micro-enlèvements d'utilisation ne permettent pas d'identifier la matière d'œuvre travaillée, par contre leur cinématique associée à la forme et à la dimension du bec biseauté laisse supposer des actions sur des matières dures organiques pour réaliser des travaux de raclage intense qui demandent une certaine précision.

Chronologiquement, le site de Bernières-sur-Seine « Les Fondriaux " indique un rattachement au Chasséen septentrional. Aucune pièce de ce type n'a pour le moment été identifiée au Cerny et au Néolithique ancien dans la région. Par contre, l'occupation Néolithique récent-final de Gaillon "La Garenne », fouillée en 2008, a livré une pièce très semblable (Prost et al., 2012, fig. 40, $\mathrm{n}^{\circ} 1$ ). Elle s'ajoute à celles de Bettencourt-Saint-Ouen dans la Somme à cette période. La concentration de mobilier de Coupvray «La Mézière-

1. In HAL Inrap. 

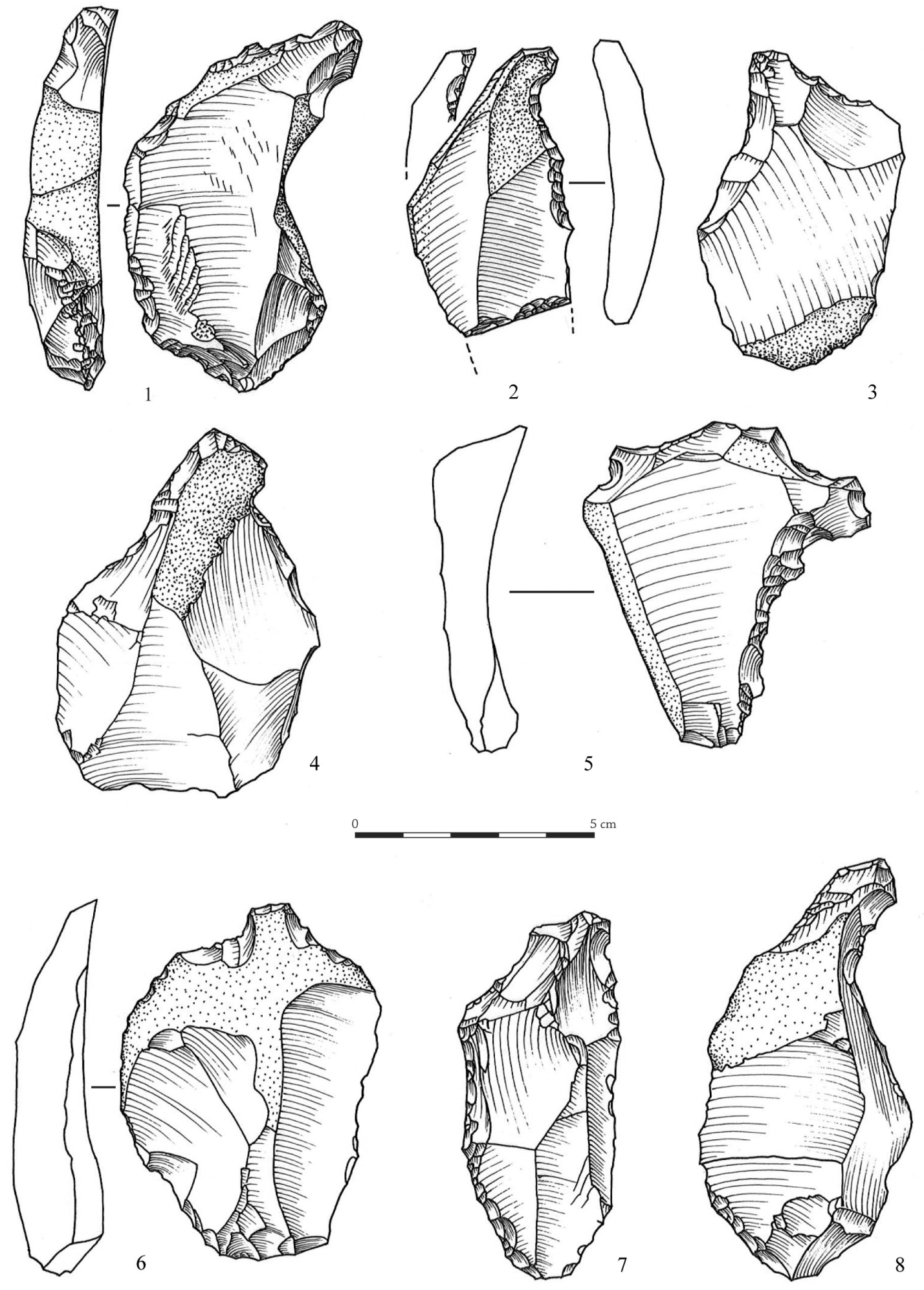

Figure 1 : Les becs biseautés : $\mathrm{n}^{\circ} 1$, Gaillon « La Garenne » (27); $\mathrm{n}^{\circ}$ 2, Bettencourt-Saint-Ouen (80); $\mathrm{n}^{\text {os }} 3,4$ et 8, Coupvray « La Mézièresud » (id. 301) Marnes-la-Vallée (77); $\mathrm{n}^{\text {os }} 5$ à 7, Bernières-sur-Seine « Les Fondriaux » (27) (dessin et DAO D. Prost, $\mathrm{n}^{\text {os }} 1,3$ à 7 ; dessin S. Lancelot et D. Prost, $\mathrm{n}^{\circ}$ 2).

Figure 1: Bevelled becs: $n^{\circ}$ 1, Gaillon "La Garenne" (27); no 2, Bettencourt-Saint-Ouen (80); $n^{o s}$ 3, 4 et 8, Coupvray "La Mézière-sud" (id.301) Marne-la-Vallée (77); $n^{o s} 5$ à 7, Bernières-sur-Seine "Les Fondriaux" (27). 
Sud » ayant livré un racloir à encoches (Prost, 1992, planche photo $\mathrm{n}^{\circ} 1$ ) pourrait indirectement dater ces becs biseautés de la même période. Ces outils, pour le moment, seraient donc présents dans le Nord-Ouest, au Néolithique moyen II et au Néolithique final.

Malgré la très faible série de sites auxquels nous nous référons, sur le plan géographique, la présence de ces pièces en Seine-et-Marne, dans la Somme et dans l'Eure laisse présager une extension géographique qui pourrait recouvrir tout ou partie du Bassin parisien, voire au-delà ou au contraire se localiser dans des territoires spécifiques donnés.

\section{Les burins transversaux}

On doit ce terme typologique à E. Vignard en 1929 lors de sa publication de l'industrie lithique aurignacienne de Nag-Hamadi en Égypte (Vignard, 1929). D’après Brézillon (Brézillon, 1971), il aurait employé pour la première fois le terme "transversal " pour désigner un type de burin comportant un mode d'aménagement différent auquel il a consacré un article en 1934 (Vignard, 1934). Après lui dans les années 1950, en France, d'autres auteurs comme D. de Sonneville-Bordes, J. Perrot et G. Laplace-Jauretche, ont repris ce terme en précisant que les burins transversaux se caractérisent par une troncature concave ou une encoche latérale d'où part le coup de burin. Le burin transversal fut reconnu comme un burin à part par l'équipe de J. Tixier qui l'a officialisé en 1980 (Tixier et al., 1980), montrant explicitement que le coup de burin transversal part essentiellement d'une encoche latéro-distale aussi bien sur lame que sur éclat (Tixier et al., 1980, fig. 23, nos 5 et 6).

C'est précisément ce type de burin que nous avons eu l'occasion de découvrir sur le site néolithique de BettencourtSaint-Ouen dans la Somme (Prost in Martin et al., 1996, fig. 220, nos 6 à 10). Ces outils étaient, de façon exceptionnelle, particulièrement nombreux. Sur les 142 burins identifiés, nous en avons décompté 54 (Prost in Martin et al., 1996, fig. 218). Depuis la fouille de ce site, très rares sont les sites néolithiques à avoir - semble-t-il - fourni ce type d'outils. Nous comptons trois sites en Normandie orientale : celui de Saint-Samson-de-la Roque dans l'Eure (Lefèbvre et Prost, 2016, fig. 25, $n^{\circ} 3$ ), celui de Bardouville « Le Moulinà-Vent » diagnostiqué par Claire Beurion (Prost in Beurion, 2013, fig. 13, no 5) et celui de Colleville "Chemin-duParc » (diag. : juin 2019; RO : Claire Beurion) qui vient d'être diagnostiqué à l'heure où nous rédigeons cet article, en Seine-Maritime. Les trois burins issus de ces nouveaux sites sont très comparables à ceux provenant de BettencourtSaint-Ouen (fig. 2). Nous rappelons les données issues de l'étude des burins de Bettencourt-Saint-Ouen que nous avions entreprise à l'époque. Ils ont pour support essentiel- lement des éclats entiers, et dans une moindre proportion, des fragments d'éclats. Plus rares sont les burins transversaux sur lame (1 pièce).

Grâce à leur fréquence sur ce site, nous avons pu effectuer des analyses statistiques (que nous reproduisons dans les figures 3, 4 et 5) confrontant ces burins à ceux non transversaux dans le but de définir avec plus d'acuité leurs caractéristiques morphotypologiques. Par exemple, l'histogramme de fréquence de la longueur des burins (fig. 3) met nettement en évidence deux courbes de Gauss distinctes entre les burins transversaux et ceux non transversaux, les supports de ces derniers étant plus allongés (pic dans la classe 60-69 cm, soit 24 pièces) que les précédents (pic dans la classe $50-59 \mathrm{~cm}$, soit 18 pièces). La courbe de fréquence de la part du cortex sur le support des burins transversaux montre des différences par rapport aux burins non transversaux (fig. 4) : la proportion des supports non corticaux est un peu plus élevée que celle des burins non transversaux. Par contre, ces derniers affichent une proportion plus élevée de produits corticaux dans la classe $<30 \%$ et 30-60\%. Au-delà de ces contingences, la position du cortex sur le support est similaire (latéral droit ou gauche, latéro-distal) sauf en position strictement distale où les burins transversaux sont nettement plus élevés. L'histogramme de fréquence sur le mode de préparation de la technique du coup de burin montre clairement que les burins transversaux se démarquent très nettement de ceux non transversaux par l'aménagement initial d'une encoche sur le bord latéral, voire d'une retouche directe, partielle (fig. 5). C'est cette particularité technologique qui les caractérise le mieux et qui, à titre de rappel, avait été retenue par les anciens auteurs pour les définir.

Pour le moment et en se fiant aux découvertes actuelles, on peut résumer les caractères morphotypologiques de ces outils. Ils ont donc presque exclusivement comme supports des éclats larges non ou peu corticaux. Leur longueur varie entre 4 et $9 \mathrm{~cm}$, le plus grand nombre entre 5 et $6 \mathrm{~cm}$. Leur largeur, quant à elle, varie de 3 à $6 \mathrm{~cm}$ en moyenne. La technique du coup de burin est toujours la même. Elle consiste à aménager localement sur un bord épais une délinéation concave par une retouche abrupte partielle ou une encoche large, directe, localisée sur la partie latéro-distale de l'éclat. Le coup de burin part de cette retouche dont le pan enlève tout ou partie de l'extrémité distale du support. On notera au passage que le pan est dit "décalé ", débordant sur la face supérieure (fig. 2, $\mathrm{n}^{\text {os }} 1,4,6$ et 7) ou inférieure (fig. 2, $\mathrm{n}^{\circ} 5$ ) voire les deux à la fois (fig. $2, \mathrm{n}^{\circ} 3$ ), selon les cas. Rares sont les coups portés perpendiculairement au bord distal qui pourraient apparaître, par leur rareté, comme un accident (fig. $2, \mathrm{n}^{\circ} 2$ ). Le coup de burin peut être simple (fig. $2, \mathrm{n}^{\text {os }} 1$, 2, 4 et 7) mais aussi double, les deux coups étant opposés, les pans se chevauchant (fig. 2, nos 3,5 et 6 ). Dans ce dernier 

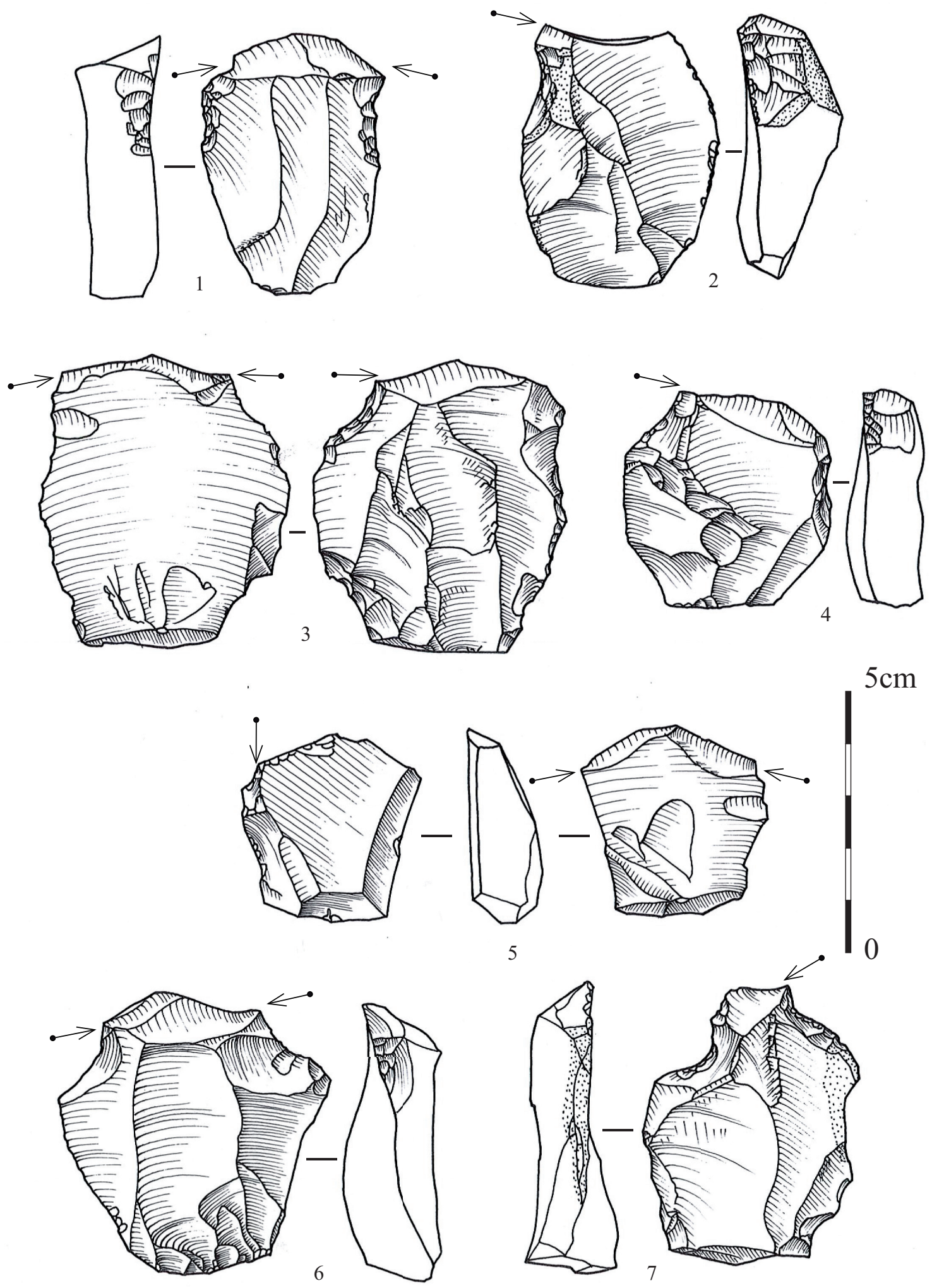

5
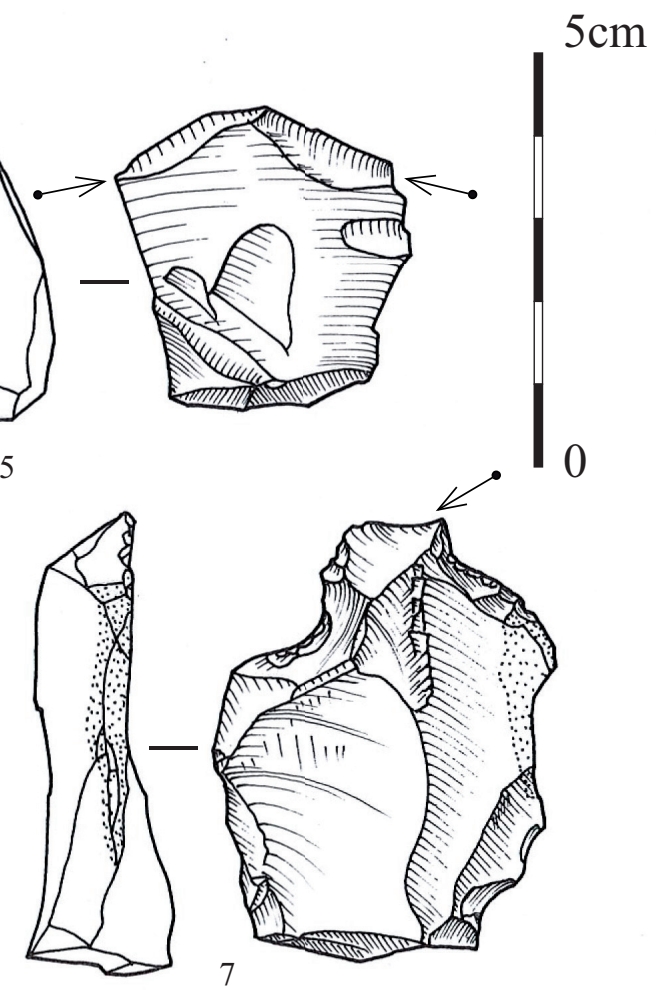

Figure 2 : Burins transversaux : $\mathrm{n}^{\text {os }} 1,2,4,6$ et 7, Bettencourt-Saint-Ouen $(80) ; \mathrm{n}^{\circ} 3$, Bardouville « Le Moulin-à-Vent » (76); $\mathrm{n}^{\circ} 5$, SaintSamson-de-la-Roque «Le Castel » (27) (dessin S. Lancelot et D. Prost, $\mathrm{n}^{\text {os }}$ 1, 2, 4, 6 et 7; dessin D. Prost, $\mathrm{n}^{\text {os }} 3$ et 5).

Figure 2: Transverse burins: $n^{o s}$ 1, 2, 4, 6 et 7, Bettencourt-Saint-Ouen (80); n 3, Bardouville "Le Moulin-à-Vent" (76); n 5, Saint-Samsonde-la-Roque "Le Castel" (27). 

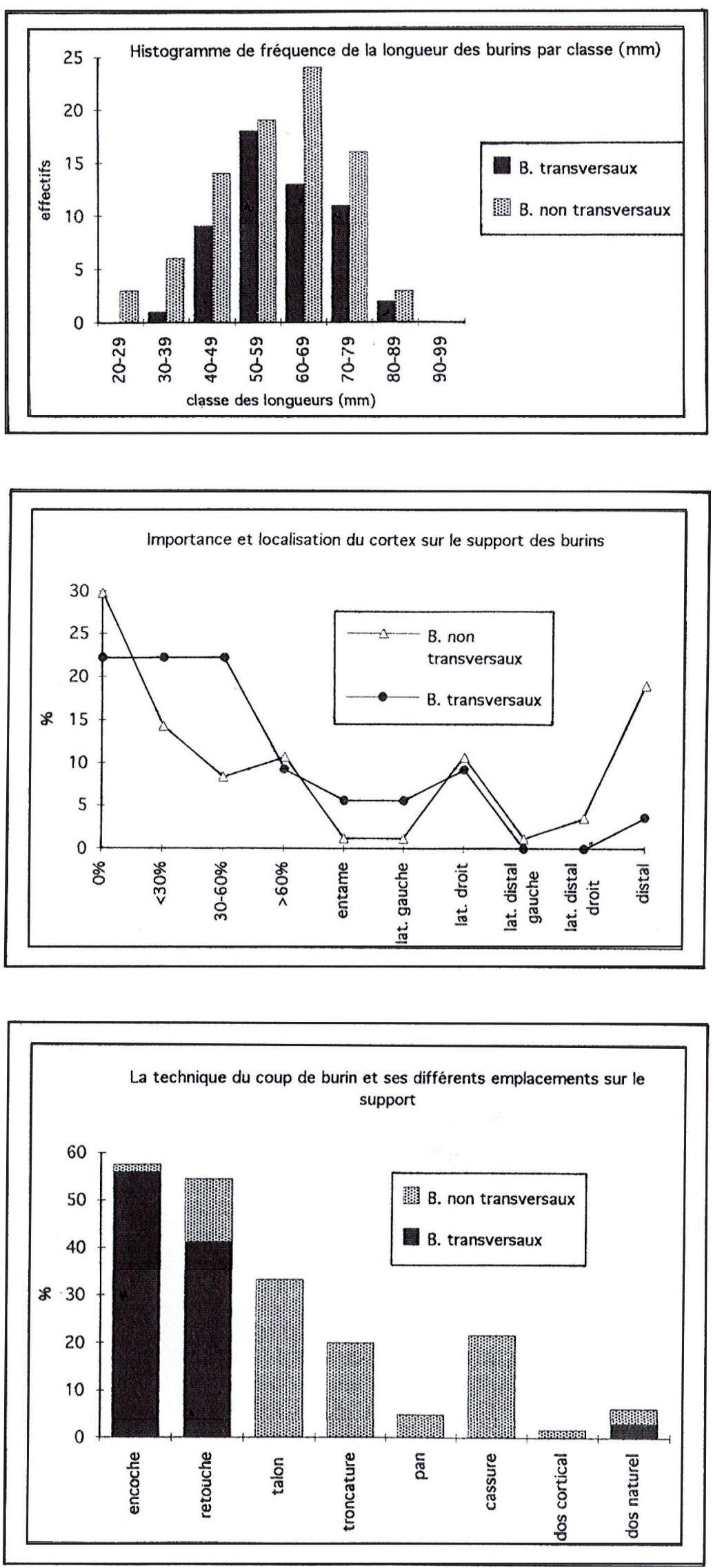

Figure 3 : Histogramme de fréquence de la longueur des burins par classe de Bettencourt-SaintOuen $(\mathrm{mm})$, ancienne figure 222, Martin et al., 1996 (DAO D. Prost).

Figure 3: Frequency histogram of burins by length, from Bettencourt-Saint-Ouen ( $\mathrm{mm}$ ), old illustration 222, Martin et al., 1996.

Figure 4 : Courbe de fréquence du cortex sur le support des burins transversaux et non transversaux de Bettencourt-Saint-Ouen, ancienne figure 223, Martin et al., 1996 (DAO D. Prost). Figure 4: Frequency curve of cortex on transverse burins support and no transverse from BettencourtSaint-Ouen, old illustration 223, Martin et al., 1996.

Figure 5 : Histogramme de fréquence des différentes techniques du coup de burin et ses différents emplacements sur le support des burins transversaux et non transversaux de Bettencourt-SaintOuen, ancienne figure 224, Martin et al., 1996 (DAO D. Prost).

Figure 5: Frequency histogram of different techniques of burin blows and different positions on transverse and no transverse burins from Bettencourt-SaintOuen, old illustration 224, Martin et al., 1996. 
cas, la délinéation que forme le fil du tranchant est particulière, de forme convexe ou sinueuse. On note enfin, cas plus rares, des coups de burin simples où il existe une encoche ou retouche opposée à la première qui pourrait s'expliquer par une volonté de contrôler la longueur du pan (fig. 2, n 7 ).

On pourrait, à l'occasion, faire des rapprochements avec les tranchets dont le coup du tranchet est technologiquement le même que le coup du burin transversal, à la grande différence près que, sur ces outils, le coup du tranchet part rarement d'une encoche. Les bords latéraux des tranchets ont fait l'objet d'une retouche continue abrupte, directe et souvent inverse, plate, pour régulariser leur forme en vue d'être emmanchés, ce qui n'est pas le cas des burins transversaux de Bettencourt qui devaient très probablement être tenus directement en main. Par ailleurs, le support des burins transversaux conserve le plus souvent un talon et un bulbe proximal (fig. 2), ce qui est rarement le cas des tranchets dont le support est différent, le talon absent, supprimé ou aminci.

Archéologiquement, le site de Bettencourt qui a fourni un nombre exceptionnel de burins transversaux est-il une exception à la règle? On pourrait en effet l'envisager. Mais deux sites normands ont livré, par la suite, deux autres burins transversaux. Bien que rares, ces sites incitent toutefois à penser à une extension géographique en Normandie de ces outils. Bien que mal datés, ces sites normands contiennent des indices qui pourraient rattacher les burins transversaux à la même période que Bettencourt-Saint-Ouen, à savoir, le Néolithique final, voire le début du Bronze ancien. Sur le plan fonctionnel, nous ignorons encore leur usage. On peut toutefois souligner, au vu de leurs différences et des traces d'utilisation que certains possèdent, le fait qu'ils étaient destinés à des actions distinctes des burins non transversaux, hypothèse qu'il faudra vérifier à l'avenir.

\section{Les couteaux demi-lune}

Nous avons identifié comme telle une pièce inédite provenant du site du Néolithique récent-final de Gaillon « La Garenne » dans l'Eure (Prost et al., 2011, fig. 8, no 6). Le fait d'avoir trouvé, peu de temps après, une pièce quasi identique et datée de la même période à Saint-Samson-de-la-Roque (Lefébvre et Prost, 2016, fig. 20, $\mathrm{n}^{\circ}$ 4) laissait supposer que nous avions affaire à un nouveau morphotype spécifique. La similarité de ces deux pièces est surprenante, aussi bien par leur forme, leurs dimensions, leur mode de façonnage et leur support (fig. 6). Ces outils ont été retouchés avec soin, leur donnant une forme symétrique. Ils présentent tous deux un dos courbe en forme de demi-lune, obtenu par retouches bifaciales longues, plates à semi-abruptes. Ce bord retouché est opposé à un tranchant rectiligne, la partie supposée active. Les supports sont des éclats minces de plein débitage.
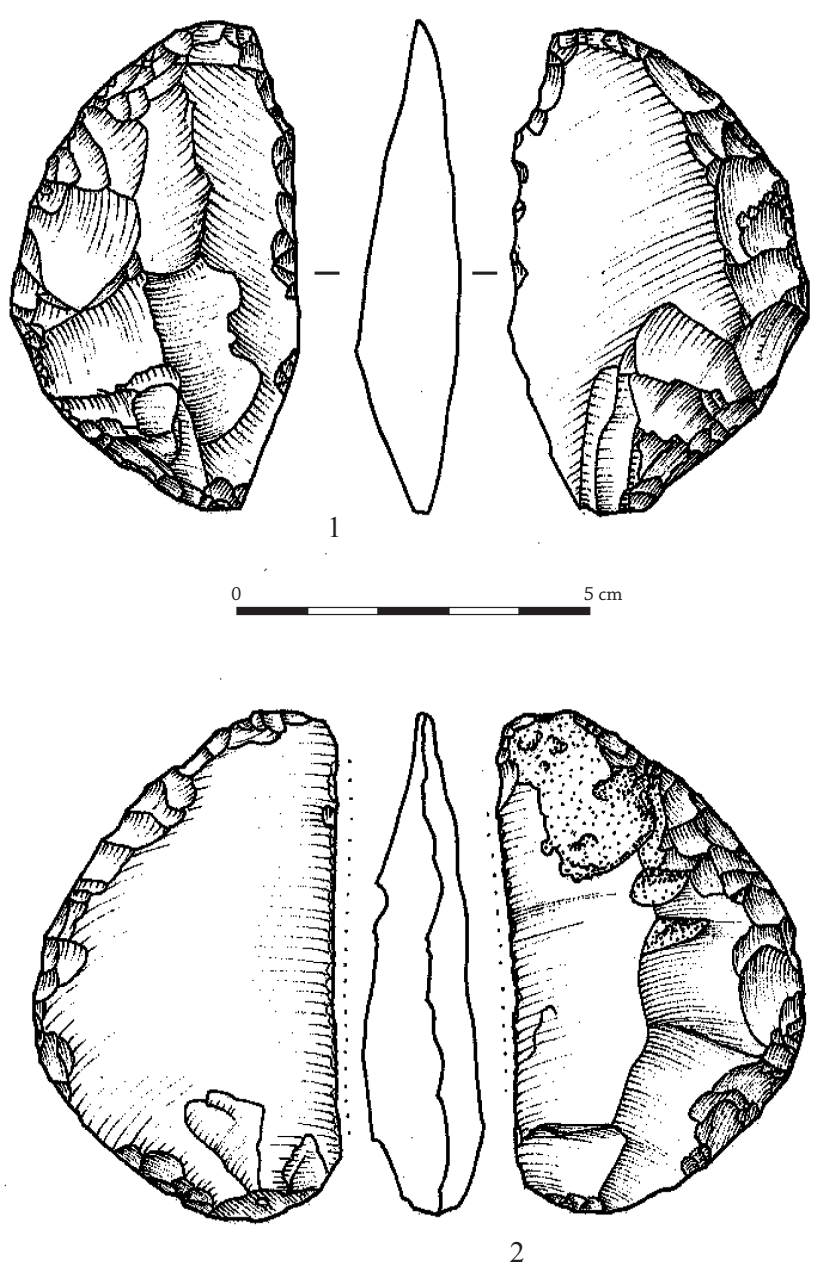

Figure 6 : Couteaux demi-lune : $\mathrm{n}^{\circ} 1$, Saint-Samson-de-la-Roque "Le Castel »; n 2, Gaillon «La Garenne » (27) (DAO D. Prost). Figure 6: Half moon knife: $n^{\circ}$ 1, Saint-Samson-de-la-Roque "Le Castel"; no 2, Gaillon "La Garenne" (27).

Leur longueur est quasiment la même, autour de $7 \mathrm{~cm}$. Il en est de même pour leur largeur médiane qui est de $4 \mathrm{~cm}$. Tous ces indices, leur morphologie et leur typométrie quasi identiques, suggèrent qu'ils ont été utilisés emmanchés selon des modalités semblables, la retouche bifaciale ayant probablement eu pour fonction d'amincir et de donner au bord une morphologie standard répondant aux normes du manche.

Létude tracéologique à faible grossissement de la pièce de Gaillon " La Garenne " met en évidence des micro-enlèvements sur les deux faces en position alternante du tranchant opposé au dos retouché. Ces micro-enlèvements, produits par frottement sur matière tendre, sont de type $\gamma$ à encoches communes, très courts, accompagnés d'un lustré brillant, formant une délinéation du fil du tranchant plus ou moins en dent de scie (fig. 7). Ces microtraces caractérisent une action prolongée sur matière organique en découpe (per- 


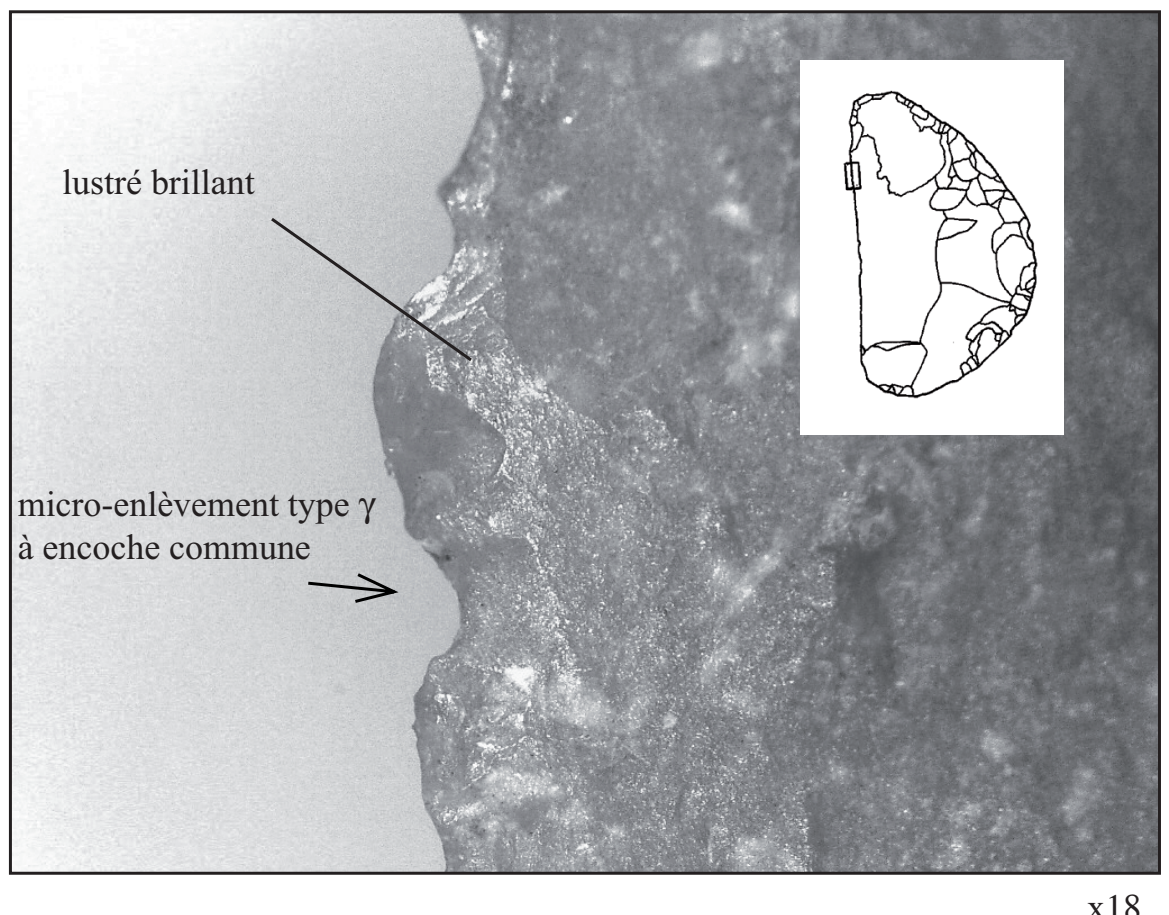

Figure 7 : Traces d'utilisations observées à la loupe binoculaire sur le couteau demi-lune de Gaillon «La Garenne » (27) (cliché et DAO D. Prost).

Figure 7: Uses-wear examined under microscope on a half moon knife from Gaillon "La Garenne" (27).

cussion posée longitudinale). Deux autres outils de nature semblable provenant de Saint-Samson-de-la-Roque ont également en commun un dos courbe à retouches bifaciales opposé à un tranchant actif, plus ou moins rectiligne (fig. 8). Comme eux, ils ont pour support des éclats de plein débitage. Leur longueur est identique, $8 \mathrm{~cm}$, mais plus grande de $1 \mathrm{~cm}$ que les deux précédents; leur largeur de 4 à $4,3 \mathrm{~cm}$, par contre, est très semblable aux précédents. Le dos est façonné par des retouches, courtes, directes, semi-abruptes et des retouches inverses plates, longues à envahissantes. Sur l'une des pièces (fig. $8, \mathrm{n}^{\circ} 1$ ), les extrémités proximales et distales ont également été aménagées par une retouche directe. Leur forme générale est moins conforme à celle en demi-lune des précédents mais s'en rapproche beaucoup. De même, des microtraces d'utilisation sur le tranchant actif sont visibles. On observe également sur la face supérieure de l'un d'eux des traces parallèles de lustré brillant qui partent du tranchant actif qui suppose cette fois-ci des actions de percussion posée transversale (fig. $8, \mathrm{n}^{\circ} 1$ ). Sur la face inférieure du tranchant actif de l'autre couteau apparaissent également des micro-enlèvements d'utilisation et une encoche dont l'action n’a pas été définie (fig. 8, n² 2).

Par leur forme en demi-lune et leur dos à retouche bifaciale, ils different en ce sens des couteaux à dos courbe fréquents au Chasséen septentrional. Ces quatre outils, datés du Néolithique final, se localisent pour le moment dans le département de l'Eure.

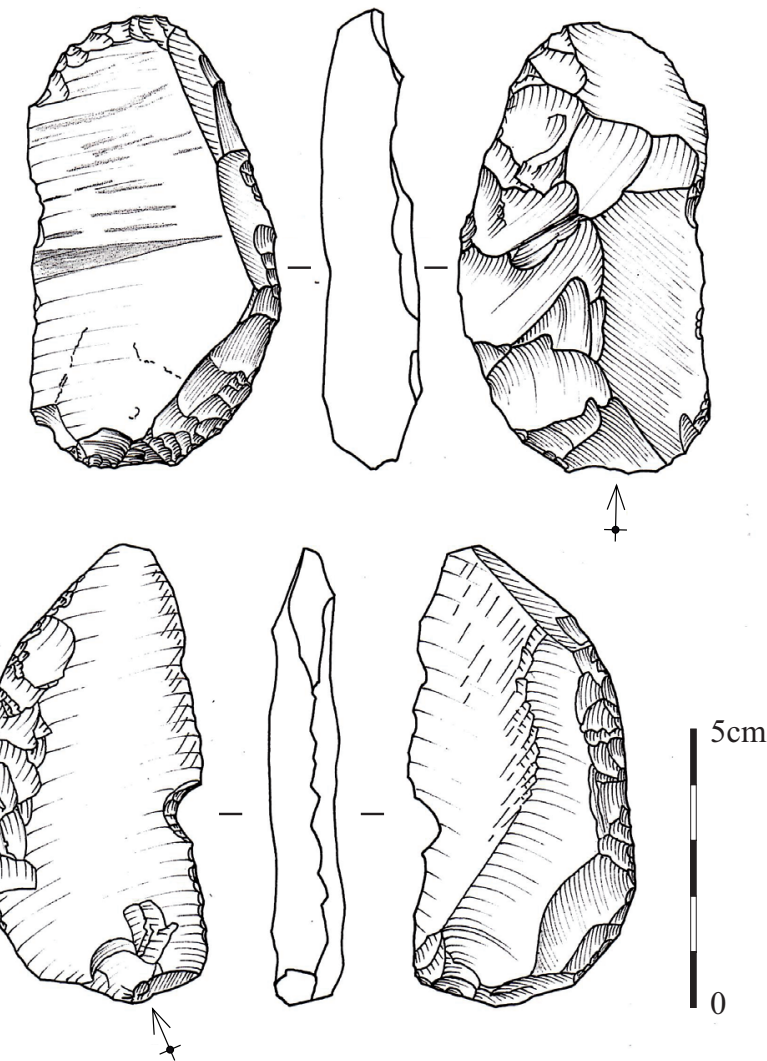

Figure 8 : Autre exemple de deux couteaux demi-lune de SaintSamson-de-la Roque «Le Castel " (dessin et DAO D. Prost).

Figure 8: Another example of two half moon knives from SaintSamson-de-la Roque "Le Castel". 


\section{Les couteaux quadrangulaires}

Brézillon nous apprend que ce terme fut pour la première fois utilisé par D. Peyronie pour décrire des couteaux allongés de forme quadrangulaire, provenant de la grotte paléolithique de la Ferrassie, comportant un dos large et brut s'opposant à un tranchant pouvant comporter des traces d'utilisation (Peyronie, 1934 cité par Brézillon, 1971). Par cette définition, nous ne sommes pas très loin de celle que l'on donnera ultérieurement à un outil du Néolithique. Classés comme outils indéterminés à l'époque, sur le site néolithique de Bettencourt-Saint-Ouen (Martin, 1996, fig. 216, $\mathrm{n}^{\circ} 2$ ), pour la première fois nous avons identifié deux pièces récoltées dans deux secteurs distincts (Q19/1 et Q11/11) qui avaient retenu notre attention par leur forme "rectangulaire " similaire. Leur ressemblance ne se limitait pas à cette forme géométrique régulière : même type de support, un tranchant actif opposé à un dos épais mais non retouché, dimensions similaires. Nous soulignions le fait que nous n'avions, à ce moment-là, aucune référence typologique pour définir ce type d'outils du Néolithique (Prost in Martin, 1996, p. 71). Un nouvel exemplaire très similaire fut découvert, par la suite, lors de la fouille de l'occupation Néolithique final-Bronze ancien de Guerny « Le Bois-Madame » en 1998-1999 (Martinez et Prost, 1999). La forme rectangulaire était quasi parfaite (Martinez et Prost, 1999, fig. $37, \mathrm{n}^{\circ} 1$ ). Se caractérisant cette fois-ci par un dos retouché, il fut enregistré parmi les couteaux et pièces à dos (Martinez et Prost, 1999, p. 31). Nous n'avons toutefois pas manqué de souligner l'étroite similitude de forme et de dimensions qu'il y avait avec ceux de BettencourtSaint-Ouen (Martinez et Prost, 1999, p. 37). D'autres couteaux de même forme rectangulaire que les précédents ont été par la suite reconnus sur le site de Mesnil-Esnard "Les Hautes-Haie » fouillé en 2005 par l'équipe de N. Fromont (Fromont et al., 2006). L. Juhel, chargé de l'étude du mobilier lithique, qui mit en évidence ces deux couteaux, n’hésita pas à remarquer l'étroite correspondance qu'il y avait à faire avec les pièces de Bettencourt-Saint-Ouen et de Guerny (Fromont et al., 2013, fig. 12). Reconnaissant l'importance typologique de ce type d'outils, les auteurs proposèrent de les désigner pour la première fois sous le nom de « couteaux quadrangulaires" (Fromont et al., 2013, p. 75).

Après 2005, deux autres couteaux quadrangulaires ont été identifiés dont une pièce à Saint-Samson-de-la-Roque (Lefebvre et Prost, 2016, fig. 19, no 1 ) et l'autre de nouveau sur la commune de Mesnil-Esnard en position de rejet sur le site de l'âge du Fer « Route de Darnétal » fouillé en 2008 par W. Varin (rapport final d'opération, à paraître).

Tous ces outils ont pour support des éclats, obtenus par percussion directe au percuteur dur, plus ou moins épais (entre 1 et $2 \mathrm{~cm}$ ). Leur longueur varie entre 4,1 et $5,8 \mathrm{~cm}$ et leur largeur, plus standard, varie entre 3,8 et $4,3 \mathrm{~cm}$. Leur rapport $\mathrm{L} / \mathrm{l}$ moyen est : 5,1/4 $=1,27$. Mis à part leur forme rectangulaire, ces outils partagent d'autres points communs. Ils possèdent, en général, un dos retouché ou brut plus ou moins rectiligne qui s'oppose, comme pour les couteaux à dos classiques, par un tranchant brut, mince, également rectiligne, dépourvu de retouche d'aménagement (fig. 9). On y observe par contre plus souvent des micro-enlèvements d'utilisation. Mais ce qui différencie nettement ces outils des simples couteaux à dos, mis à part leur forme rectangulaire, c'est l'aménagement des bords proximaux et distaux du support, formant un pan rectiligne, abrupt, dégagé par retouche (fig. 9, $\mathrm{n}^{\text {os }} 1,2,3$ et 5), par coup de burin (fig. 9, $\mathrm{n}^{\circ}$ 5) ou par cassure (fig. 9, $\mathrm{n}^{\circ} 4$ ).

Malgré la très faible quantité de pièces identifiées, il est possible de dresser un premier portrait-robot de ces outils à partir de la superposition de leur contour (fig. 10).

De manière générale, on remarquera qu'ils se rapprochent typologiquement des racloirs (ou scies) à encoches. Sur le site Néolithique final de Charavines, deux pièces identifiées comme racloirs à encoches ont été nommés " racloirs ou scies à encoches peu évolués " par le fait qu'ils ressemblent beaucoup à ces outils mais sans encoches (Thevenot $e t$ al., 1976, fig. $23, \mathrm{n}^{\text {os }} 12$ et 13 ). De par leur forme rectangulaire, on peut en fait les identifier désormais comme des couteaux quadrangulaires similaires à ceux de Normandie. Cet exemple montre dans ce cas que c'est la présence/ absence des encoches qui fait la différence pour les départager. Des similitudes sont également à faire avec certains couteaux à moissonner sans encoches. Le site de Gaillon « La Garenne » nous en fournit un exemple régional remarquable sur lame (fig. 11). Cette pièce, retouchée avec beaucoup de soin possède un dos abrupt en grande partie retouché mais non rectiligne. L'extrémité distale est retouchée comme sur les couteaux quadrangulaires. Sur le tranchant actif, on observe de façon quasi continue sur la face supérieure des micro-enlèvements typiques de type $\gamma$ linéaire, face supérieure, associés à un lustré brillant face inférieure pouvant correspondre à la fonction de faucille à moissonner (fig. 11) . Sa longueur $-10,2 \mathrm{~cm}$ - est deux fois celles des couteaux quadrangulaires. Par contre, sa largeur est la même : $4 \mathrm{~cm}$. Les extrémités de la lame et le tranchant rectiligne donnent à cette pièce une forme quasi rectangulaire. La longueur, le support sur lame et la minceur de celui-ci font la différence. Cet outil semble avoir été destiné à être emmanché sur un manche en bois comme c'est le cas des couteaux à moissonner des sites des milieux lacustres circum-alpins (fig. 11). Or, ce type d'emmanchement ne parait pas approprié pour nos couteaux quadrangulaires qui apparaissent trop épais, trop courts, pour ce type d'emmanchement. Leur mor- 

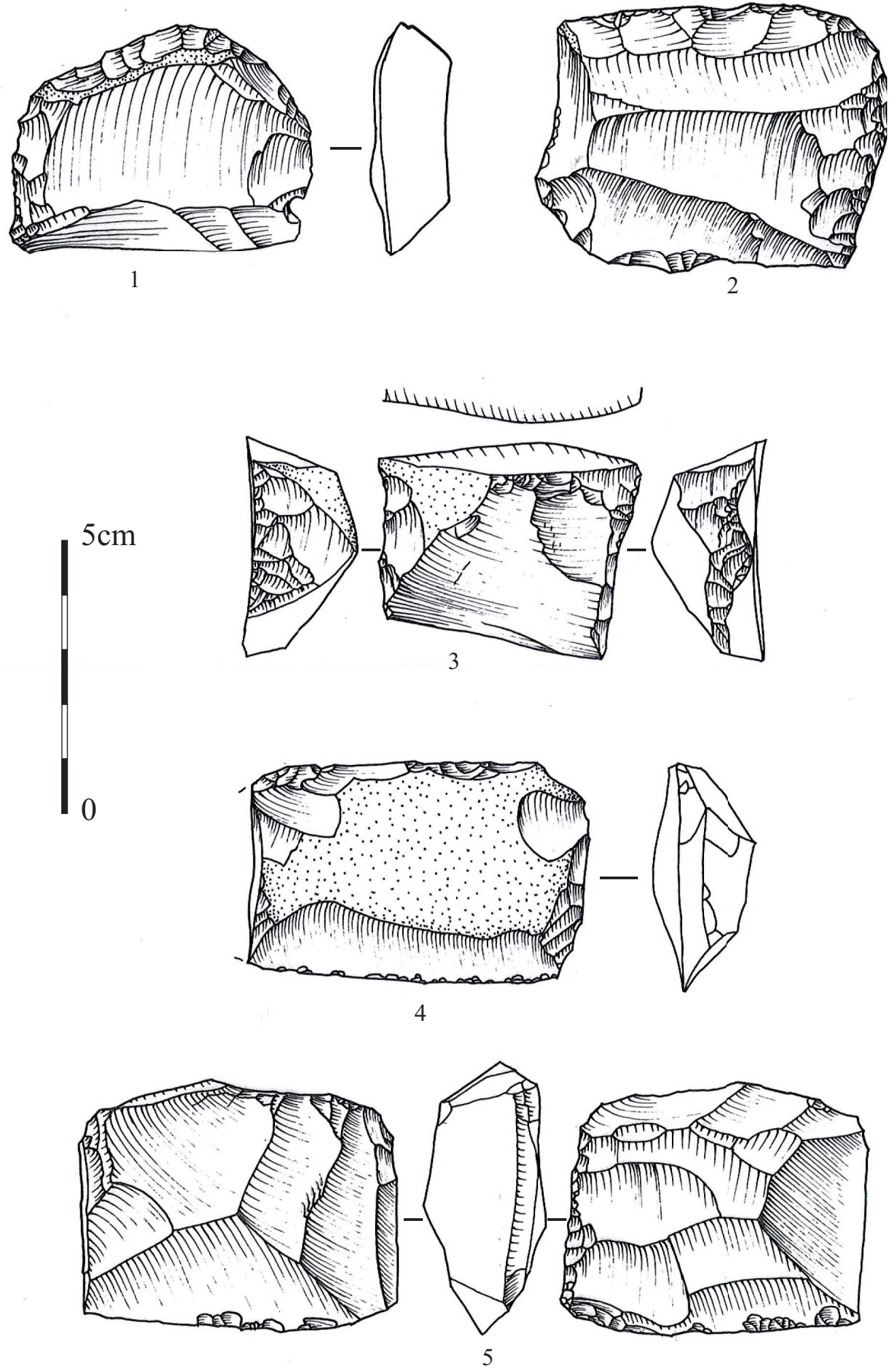

Figure 9 : Les couteaux quadrangulaires : $\mathrm{n}^{\circ}$ 1, Mesnil-Esnard « Route de Darnétal » (76); no 2, Saint-Samson-de-la Roque « Le Castel » (27); $\mathrm{n}^{\circ}$ 3, Mesnil-Esnard « Les Hautes-Haies » (76); n 4, Guerny «Le Bois-Madame » (27); n 5, Bettencourt-Saint-Ouen (80) (dessin D. Prost, $\mathrm{n}^{\text {os }} 1$ à 4 ; dessin S. Lancelot et D. Prost, $\mathrm{n}^{\circ}$ 5).

Figure 9: Quadrangular knives: $n^{\circ}$ 1, Mesnil-Esnard "Route de Darnétal" (76); no 2, Saint-Samson-de-la-Roque "Le Castel" (27); n' 3, MesnilEsnard "Les Hautes-Haies" (76); no 4, Guerny "Le Bois-Madame" (27); n 5, Bettencourt-Saint-Ouen (80). 

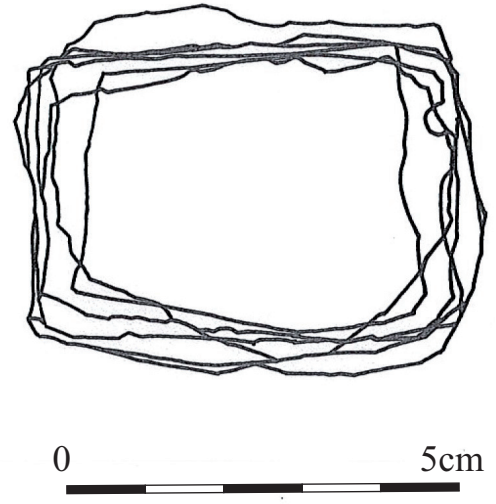

Figure 10 : Portraitrobot des couteaux quadrangulaires (dessin et DAO D. Prost).

Figure 10: Identikit picture of quadrangular knives. phologie semble toutefois conforme à un mode d'emmanchement mais distinct des précédents couteaux, destinés à une fonction ou une action différente. D'un point de vue chronologique, les sites les mieux datés sont BettencourtSaint-Ouen (Néolithique final, phase campaniforme) et Guerny "Le Bois-Madame " (transition Néolithique finalBronze ancien). Ces couteaux quadrangulaires se rattacheraient donc à la deuxième moitié du III ${ }^{e}$ millénaire. Le site de Mesnil-Esnard qui s'est référé à ces deux précédents sites pour dater son mobilier lithique, et donc ses couteaux quadrangulaires, est daté avec précaution par ses auteurs dans une tranche plus large qui couvrirait le III $^{e}$ millénaire avec comme option possible le Néolithique récent-final. Enfin, l'occupation principale de Saint-Samson-de-la-Roque, à laquelle appartient le couteau quadrangulaire, se situe dans la même problématique que celle de Mesnil-Esnard.

C'est donc au III ${ }^{e}$ millénaire et jusqu'au début du $\mathrm{II}^{e}$ millénaire que pourrait se situer chronologiquement cet outil d'après les premiers indices en notre possession. Quant à l'extension géographique de cet outil, elle se limite pour le moment aux départements de la Somme, de la SeineMaritime et de l'Eure.

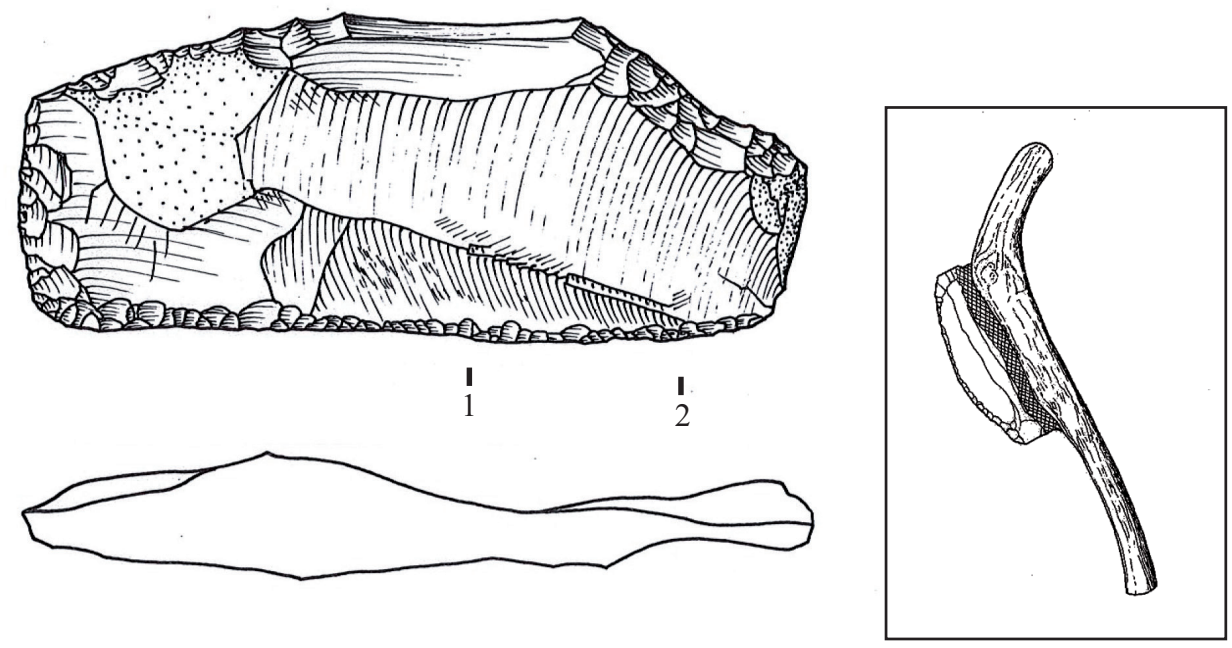

cliché 1

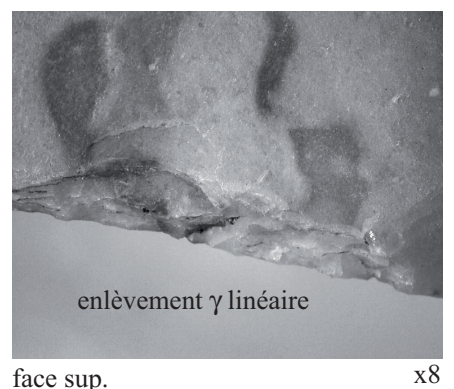

$\mathrm{x} 8$ cliché 2

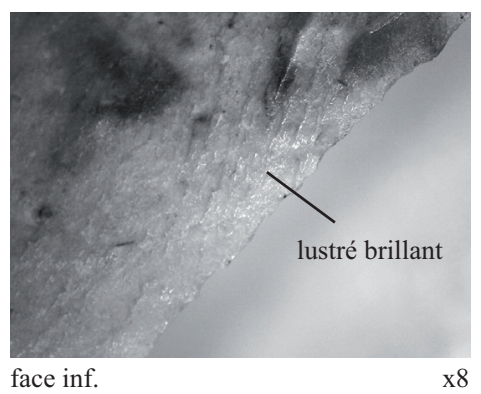

Figure 11 : Grand couteau à moissonner sur lame de Gaillon "La Garenne " (27); microenlèvements de type $\gamma$ linéaire face supérieure et lustré brillant face inférieure observés à la loupe binoculaire; exemple d'emmanchement de couteau à moissonner sur lame, Egolzwil 5 (Lucerne, Suisse), du Néolithique moyen (Pétrequin, 1984, d'après cliché 16, Musée national suisse) (dessin, cliché tracéo et DAO D. Prost).

Figure 11: Large harvest knife on blade from Gaillon "La Garenne" (27); use-wear type linear $\gamma$ on upper side and glossy luster on lower side under microscope; fitting of a handle, example of harvest knife on blade, Egolzwil 5 (Lucerne, Suisse), middle Neolithic (Pétrequin, 1984, photo 16, musée national suisse). 


\section{Les pointes laminaires à coches basales}

On dénombre cinq pièces provenant de quatre sites. La plus ancienne découverte archéologique provient du site de Bettencourt-Saint-Ouen dans la Somme, (Martin, 1996). À cette époque, nous avions rangé la pièce dans la catégorie " poignard » et avions remarqué qu'elle possédait à ses extrémités des traces de bord émoussé caractéristique des pierres à briquet, ayant été interprété comme acte de réemploi, (Prost in Martin, 1996, p. 63 et fig. 214, n 6). Spatialement, elle était accompagnée d'un autre poignard entier qui possédait les mêmes traces d'utilisation à son extrémité proximale, marquée cette fois-ci par une seule coche basale. Poignard ou pierre à briquet? Il était difficile à l'époque de trancher et que dire des coches basales? Tant que nous n'avions pas trouvé de référence régionale sûre de pierres à briquet associées à des coches basales, la question de la fonction de ces coches restait en suspens. Il a fallu attendre, quelques années plus tard, la mise au jour de quatre autres pièces similaires en Normandie pour envisager que ces outils à coches basales n'étaient pas, en première instance, des pierres à briquet. Deux pièces proviennent de l'ensemble $18 \mathrm{a} / \mathrm{d}$ du site de Saint-Vigor-d'Ymonville en Seine-Maritime, fouillé sous la direction de Cyril Marcigny (Marcigny, 2002, fig. 179, $\mathrm{n}^{\text {os }} 4$ et 6), une autre du site de Gaillon "La Garenne ", dans l'Eure, fouillé sous la direction de l'auteur de cet article (Prost et al., 2011, fig. 8, no 4) et la dernière, du site prospecté par G. Lefèbvre à Saint-Samson-de-la-Roque, également dans l'Eure (Lefèbvre et Prost, 2016, fig. 23).

En plus de leurs coches basales, ces outils ont en commun des lames comme supports. Elles ont toutes un profil rectiligne voire légèrement arqué et une largeur similaire (entre 2,5 et 2,9 cm). Quatre d'entre elles comportent, face supérieure, trois pans principaux à arêtes plus ou moins parallèles. La direction des enlèvements antérieurs traduit un débitage unidirectionnel exclusif sur trois d'entre elles (fig. 12, $\mathrm{n}^{\text {os }} 1,2$ et 4 ), préférentiel sur les deux autres, avec un recours partiel au débitage bidirectionnel opposé (fig. 12, $\mathrm{n}^{\text {os }} 3$ et 5 ). Ces lames, produites probablement par percussion directe au percuteur tendre, se terminent toutes en pointe par une retouche directe, latérale et continue. Toutefois, ce petit échantillon laisse présager les prémices de deux sousmorphotypes distincts. Le premier se démarquerait par des pointes courtes (longueur inférieure à $8 \mathrm{~cm}$ ) et une retouche unilatérale gauche (fig. $12, \mathrm{n}^{\text {os }} 1$ et 2 ). Le deuxième serait représenté par des pointes longues (longueur supérieure à $8 \mathrm{~cm}$ ) et une retouche bilatérale (fig. $12, \mathrm{n}^{\text {os }} 3$ à 5 ).

Chronologiquement, les pointes de Saint-Vigord'Ymonville (fig. 12, $\mathrm{n}^{\text {os }} 2$ et 3 ) appartiennent à l'ensemble 18 a/d dont le mobilier céramique et lithique est rattaché au Chasséen septentrional (Marcigny, 2002, p. 153-
155). La pointe de Bettencourt-Saint-Ouen est attribuable au Néolithique final, phase campaniforme (Martin, 1996, p. 105). La pointe de Gaillon « La Garenne » appartiendrait, quant à elle, à une occupation plus ancienne du Néolithique final, première moitié du $\mathrm{III}^{\mathrm{e}}$ millénaire, voire à une transition Néolithique récent-final (Prost, 2012, p. 155). Pour la pièce de Saint-Samson-de-la-Roque, issue d'une prospection, l'appartenance chronoculturelle est plus incertaine, datable du Néolithique final au sens large. On notera sa grande similarité avec la pointe courte de Saint-Vigord'Ymonville - ces deux sites étant situés en baie de Seine, presque l'un en face de l'autre, sur les deux rives, à quelques kilomètres de distance.

En conclusion, ces outils sont attribuables pour le moment au Chasséen septentrional et au Néolithique final, et dans trois départements : la Somme, la Seine-Maritime et l'Eure.

\section{Les herminettes à encoches}

Nous terminons par la présentation d'un outil qui n'est pas nouveau en soi, nous allons le voir plus en détail, mais, par sa rareté sur les sites néolithiques et sa quasi-présence dans une aire géographique donnée, la Normandie orientale, restant au demeurant encore peu connu de la communauté des néolithiciens hors de notre région.

C'est au tout début du $\mathrm{xx}^{\mathrm{e}} \mathrm{s}$. que furent découverts ces premiers outils par des collectionneurs prospectant les champs labourés et zones forestières du Pays de Caux, en bord de mer. D'après Cayeux (Cayeux, 1957), c'est la station d'Yport-Froberville qui fut le premier site à avoir livré ces pièces. D'autres furent signalées en 1912 par M. Duteurtre dans la station dite du "Val-Reinette", également celle des "Sapinières " en haut du Havre, située dans la forêt de Montgeon (Duteurtre, 1912). De nouvelles découvertes furent faites à Yport sur le plateau des Hogues, notamment en 1939, puis sur les communes de Bléville ${ }^{2}$, au lieu-dit "La Corvée ", et Gommerville près de Saint-Romain-deColbosc en 1942, confirmant l'existence de ce type d'outils dans cette partie de la Seine-Maritime. Ces trouvailles ont fait l'objet de cinq publications parues - dont la majorité avant l'occupation allemande - dans les bulletins des associations locales (Dubus, 1910; Duteurtre, 1912; Cayeux, 1957). On doit ensuite la publication de deux articles signés de la main de L. Cayeux, l'érudit local, qui a eu le mérite de faire connaitre ces outils hors de la région, parus dans deux bulletins de la Société préhistorique française dont le deuxième est réservé exclusivement à ces outils sous le titre Les herminettes et les houes à encoches latérales du Précampignien

2. Cette commune n'existe plus, comme celles de Sanvic et GravilleSainte-Honorine, annexées à la ville du Havre lors de son extension au $\mathrm{xx}^{\mathrm{e}} s$. 
Figure 12 : Les pointes laminaires à coches basales : $\mathrm{n}^{\circ} 1$, Saint-Samson-de-la Roque « Le Castel » $(27) ; \mathrm{n}^{\text {os }} 2$ et 3, Saint-Vigor-d'Ymonville « Les Sapinettes - La Mare des Mares " (76); nº 4, Gaillon «La Garenne » (27); n ${ }^{\circ}$, Bettencourt-Saint-Ouen (80) (dessin D. Prost, $\mathrm{n}^{\text {os }} 1,4$ et 5 ; dessin E. Ghesquière et D. Prost, $\mathrm{n}^{\text {os }} 2$ et 3).

Figure 12: Blade points with basal notches: $n^{\circ} 1$, SaintSamson-de-la-Roque "Le Castel" (27); $n^{o s} 2$ et 3, SaintVigor-d'Ymonville "Les Sapinettes - La Mare des Mares" (76); no 4, Gaillon "La Garenne" (27); n 5, BettencourtSaint-Ouen (80).

du Pays de Caux (Cayeux, 1957), première synthèse sur le sujet et à laquelle se réfèrera plus tard M.-N. Brézillon pour sa détermination (Brézillon, 1971, p. 253). Cayeux continua à publier ponctuellement des découvertes locales de ces outils, notamment à "La Mare-Rouge ", près de la station des "Sapinières " (Cayeux, 1970), confirmant l'importance des occupations néolithiques sur le plateau nord du Havre. En conclusion, jusqu'aux années 1960, les principales découvertes provenaient du Pays de Caux, à partir de ramassages de surface, de coupes ou de sondages. Ces pièces, associées à d'autres vestiges lithiques, étaient en général attribuées au Néolithique de type Campignien et au Précampignien (Mésolithique), selon Cayeux. C'est dans la région de l'Eure et au sud-ouest de la Seine-Maritime que J. Gehenne mit au jour des herminettes à encoches lors de prospections et de recherches dans des collections locales. Il nous signala des pièces en particulier à Pîtres, Louviers « Le Buc » et Grand-Couronne "Les Essarts " près de Rouen. Au cours de sa thèse intitulée Le Néolithique en Seine-Maritime, J.-P. Watté reprit à son compte l'inventaire des découvertes
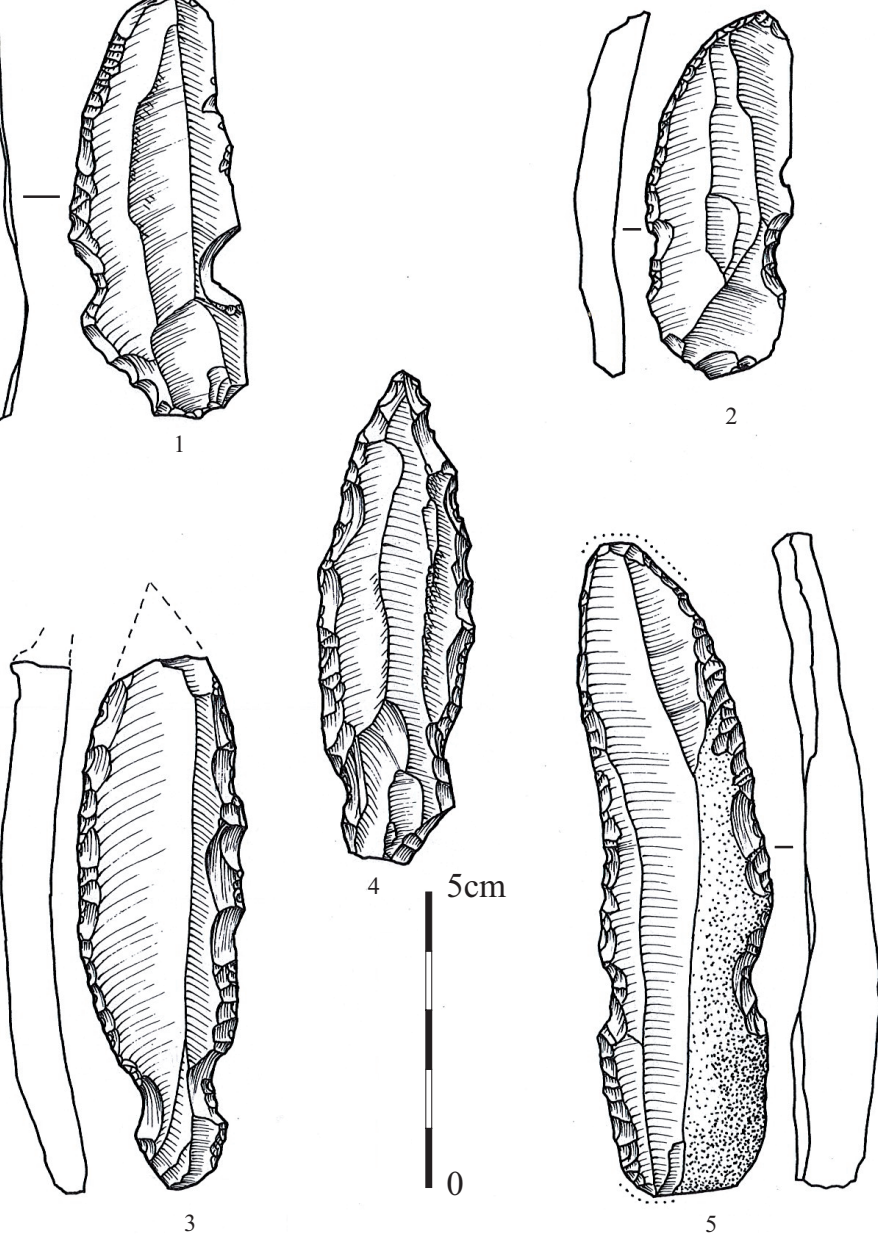

de ces outils, enrichissant la documentation de ses prédécesseurs (Watté, 1991). On apprend ainsi l'existence d'autres sites ou découvertes fortuites comme à Pommeréval, près de Neufchâtel-en-Bray. Mais c'est surtout à Yport-SaintLéonard que se concentre l'attention de ce chercheur qui réalise pour la première fois des fouilles sur ce site ${ }^{3}$, confirmant la présence de ces outils, associés cette fois-ci à de la céramique dans un contexte daté, le Néolithique récent-final (Watté, 1991, fig. 378). À la même période, les fouilles programmées de l'important site du Néolithique final menées par T. Lepert à Saint-Wandrille-Rançon (Seine-Maritime) ont fourni, de nouveau en contexte daté, une très belle herminette à encoches (Lepert, 1987, fig. 13, nº 4). Par la suite, ce sont les opérations préventives réalisées par l'Afan, puis par l'Inrap, qui mettront au jour, toujours ponctuellement et en Normandie orientale, ce type d'outils. Citons les sites de Bernières-sur-Seine "Les Fondriaux » (Prost et Biard,

3. Cf. rapport de synthèse (extrait de thèse) des comptes rendus de la fouille des années 1986, 1987 et 1988 (Watté, 1991). 
2000), Bouafles « La Plante-à-Tabac » (Prost et al., 2004 et 2005), Honguemare "ZAC du Roumois » (Honoré, 2007), Saint-Vigor-d'Ymonville " La Mare-des-Mares » (Marcigny, 2002), Le Tréport " Rue Dixon » (Kliesch, 2012), Gaillon "La Garenne » (fouille 2016) (Riche, à paraittre), etc.

Selon Cayeux, au début du $\mathrm{Xx}^{\mathrm{e}}$ s., ces outils ont reçu des appellations comme " haches à coches ", "grattoirs à encoches ", et plus modestement "pièces à encoches ". Considérant que ces outils auraient été utilisés comme herminettes, cet auteur a proposé de les nommer « herminettes à encoches latérales ", terme officialisé par M. Brézillon, mais qui, avec le temps, s'est résumé à celui d'herminettes à encoches.

Au total, à la date de 2017, plus de 50 herminettes à encoches ont été signalées provenant d'au moins 35 sites (tabl. $1^{4}$ ). Nous avons pu étudier personnellement 13 d'entre elles et observer une partie des autres à partir de dessins publiés.

Ces outils se caractérisent avant tout par leurs encoches latérales, larges et profondes, qui se situent sur la partie médiane de leur support, leur donnant une forme commune qui rappelle celle en huit ou celle d'un sablier (fig. 13). La matière première utilisée est un silex local, de plus ou moins bonne qualité selon les cas, correspondant, en général, à celui utilisé pour le reste de l'outillage. Le support est varié et fait l'objet d'un choix très opportuniste. Si on reconnaît des éclats, parmi les supports, on constate également des pièces fabriquées dans la masse probablement à partir de petits blocs, cassons ou fragments de bloc. Mais, dans la majorité de ces cas, on n'observe pas de stigmates qui permettent d'identifier nettement le type de support. Ce sont en fait, bien souvent, des produits en marge du circuit classique de la chaîne opératoire qui, de conception simple, demandaient très peu d'investissement. L'analyse typométrique portant sur 25 pièces nous indique que la longueur moyenne enregistrée est de $8,9 \mathrm{~cm}$, la largeur moyenne de $6 \mathrm{~cm}$, celle du tranchant de $5,5 \mathrm{~cm}$. On retiendra que le talon, de $4 \mathrm{~cm}$ en moyenne, est moins large que le tranchant. Il est légèrement supérieur à celui des encoches de $3,8 \mathrm{~cm}$. Enfin, on notera que ces outils sont relativement épais : 2,6 cm d'épaisseur en moyenne. Ces mesures sont quasi identiques à celles enregistrées par Cayeux : " 9 à $10 \mathrm{~cm}$ de long sur $6 \mathrm{~cm}$ de large, mesure prise au taillant "(Cayeux, 1957, p. 572). Ces valeurs moyennes ne doivent pas cacher le fait que ces outils peuvent être affectés par une variabilité typométrique parfois relativement importante (fig. 13) qui ne permet pas d'identifier des dimensions standardisées. Il est

4. La liste des sites ayant fourni des herminettes à encoches n'est pas exhaustive.
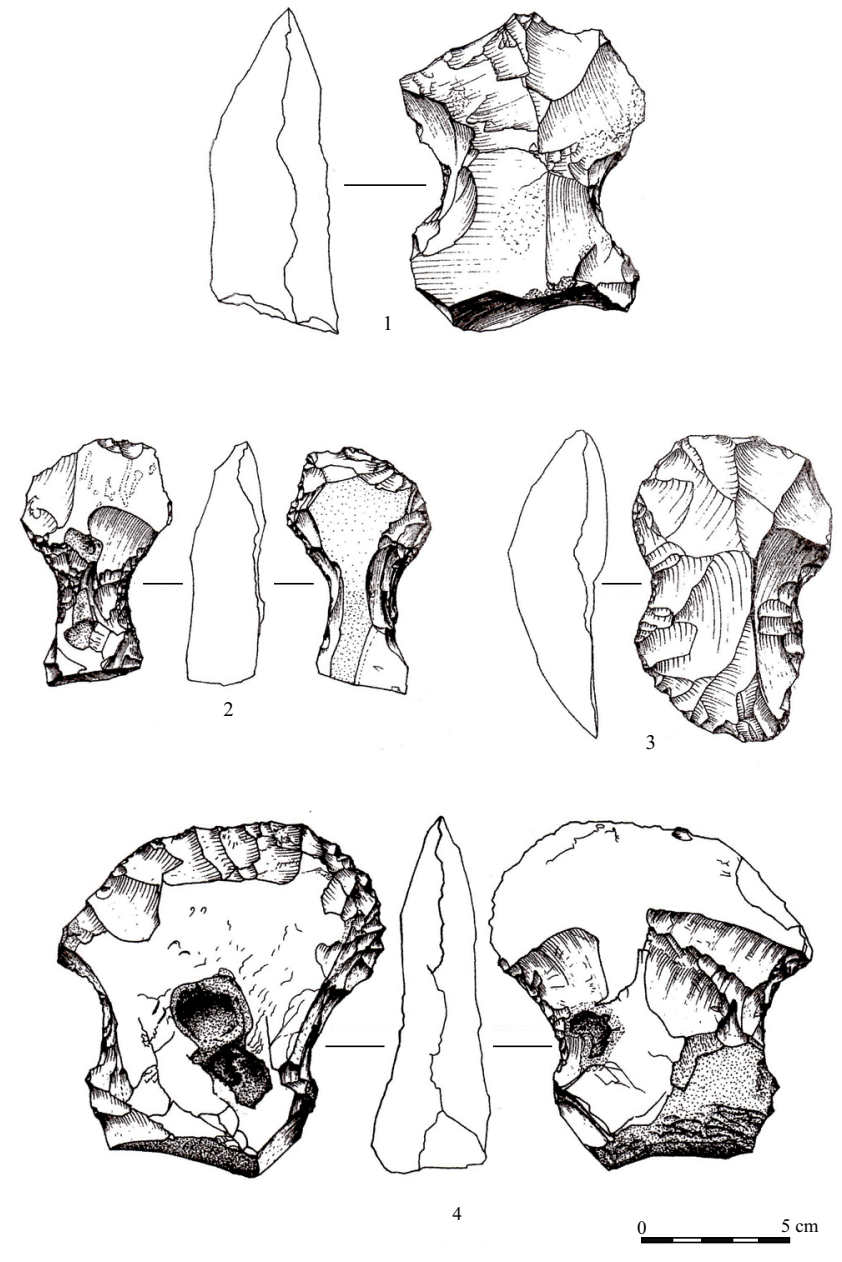

Figure 13 : Exemples d'herminettes à encoches : $\mathrm{n}^{\circ} 1$, Bouafles "La Plante-à-Tabac " (27), BVSG; n 2, Honguemare-Bosgouet, A28 (76), Néolithique moyen II ?; n 3, Saint-Vigor-d'Ymonville "Les Sapinettes - La Mare des Mares » (76) ensemble 17; n 4, Saint-Wandrille-Rançon (76), Néolithique final (dessin D. Prost, $\mathrm{n}^{\text {os }} 1,2$ et 4 ; dessin E. Ghesquière et D. Prost, $\mathrm{n}^{\circ} 3$ ).

Figure 13: Examples of notched adzes : $n^{\circ} 1$, Bouafles "La Plante-àTabac" (27), BVSG; n' 2, Honguemare-Bosgouet, A28 (76), middle II Neolithic?; $n^{\circ}$ 3, Saint-Vigor-d'Ymonville "Les Sapinettes - La Mare des Mares" (76) ensemble 17, Final Neolithic; $n^{\circ} 4$, SaintWandrille-Rançon (76), Final Neolithic.

possible toutefois d'en établir un portrait-robot (fig. 14). La superposition du contour de 12 de ces outils fait apparaître indéniablement une certaine constance morphologique, les contours se resserrant essentiellement au niveau des encoches. Ceci permet de montrer aussi de façon plus visuelle le degré de variabilité plus important qui affecte le talon par rapport au tranchant, les contours étant distribués de façon plus régulière dans ce dernier cas, comme au niveau des encoches; ce qui permet de conclure que le 


\begin{tabular}{|c|c|c|c|c|c|}
\hline Bernières/Seine & Les Fondriaux & 27 & F. préventive Afan & Prost et Biard, 2000 & SRA-Canteleux \\
\hline Bleville & La Corvée & 76 & prospection & Cayeux, 1957 & coll. Guyader \\
\hline Bosc-le-Hard & $?$ & 76 & prospection & Watté, 1990 & coll., Boulanger \\
\hline Bouafles & La Plante-à-Tabac & 27 & F. préventive Afan & Prost et al., 2005 & SRA-Canteleux \\
\hline Bouafles & carrière RD313 & 27 & diagnostic Inrap & Prost et al., 2004 & SRA-Canteleux \\
\hline Clecy & Les Anneaux & 14 & prospection & Cayeux, 1957 & coll. Cayeux \\
\hline Gaillon & La Garenne & 27 & F. préventive Inrap & Riche et al., à paraître & Grand-Quevilly \\
\hline Gommerville & $?$ & 76 & prospection & Cayeux, 1957 & coll. Cayeux \\
\hline Grande-Couronne & Les Essards & 76 & prospection & Gehenne doc. Privée & coll. Géhenne \\
\hline Honguemare-Bosgouet & A28 & 27 & diagnostic Inrap & Honoré, 2003, non publié & SRA-Canteleux \\
\hline Honguemare-Guénouville & ZAC du Roumois & 27 & diagnostic Inrap & Honoré 2007 & SRA-Canteleux \\
\hline Le Havre & La Mare-Rouge & 76 & prospection & Cayeux, 1970 & coll. Cayeux \\
\hline Le Havre & Les Sapinières & 76 & prospection & Cayeux, 1957 & coll. Cayeux \\
\hline Le Havre & Le Val-Reinette & 76 & prospection & Duteurtre, 1912 & coll. Duteurtre \\
\hline Le Havre & Le Mont-Gaillard & 76 & diagnostic & Watté, 1998 & $?$ \\
\hline Le Tréport & rue Dixon & 76 & diagnostic Inrap & Kliesch et al., 2012 & SRA-Canteleux \\
\hline Louviers & Le Buc & 27 & prospection & Gehenne, doc. privée & coll. Gehenne \\
\hline Mesnil-Esnard & route de Darnétal & 76 & F. préventive Inrap & Varin et al., à paraître & Grand-Quevilly \\
\hline Pîtres & $?$ & 27 & prospection & Géhenne, doc. Privée & coll. Gehenne \\
\hline Pomméreval & $?$ & 76 & prospection & Watté, 1990 & $?$ \\
\hline Poses & Sur la Mare & 27 & F. préventive Afan & Bostyn et al., 2003 & SRA-Canteleux \\
\hline Quitebeuf & $?$ & 27 & prospection & Watté, 2001 & $?$ \\
\hline Saint-Aubin-sur-Gaillon & $\begin{array}{l}\text { ZAC des Champs- } \\
\text { Chouettes }\end{array}$ & 27 & diagnostic Inrap & Beurion et al., 2006 & SRA-Canteleux \\
\hline Saint-Riquier-es-Plain & Le Golf & 76 & F. préventive Inrap & Lukas et al. à paraître & Grand-Quevilly \\
\hline St-Samson-de-la-Roque & Le Castel & 27 & prospection & Lefebvre et Prost, 2015 & coll. Lefèbvre \\
\hline St-Vigor-d'Ymonville & La Mare-des-Mares & 76 & F. préventive Afan & Marcigny et al., 2002 & SRA-Canteleux \\
\hline St-Wandrille-Rançon & Manoir-du-Perroy & 76 & Fouille programmée & Lepert, 1987 & SRA-Canteleux \\
\hline Sevrai & La Haute-Rivière & 61 & F. préventive Afan & Giazzon et al., 2014 & $?$ \\
\hline Yport-Froberville & station d'Yport & 76 & prospection & Huet, Grespinet, 1965 ? & coll. ? \\
\hline Yport-Froberville & Les Hogues & 76 & prospection & Dubus, 1910 & coll. Guérard \\
\hline Yport-Froberville & Les Hogues & 76 & prospection & Cayeux, 1957 & coll. Cayeux \\
\hline Yport-Froberville & Les Hogues & 76 & prospection & Cayeux, 1957 & coll. Egloff \\
\hline Yport-Froberville & $?$ & 76 & prospection & Cayeux, 1957 & coll. Guyader \\
\hline Yport-Saint-Léonard & $?$ & 76 & Fouille programmée & Watté, 1992 & coll. Watté \\
\hline
\end{tabular}

Tableau 1 : Inventaire des sites normands ayant fourni des herminettes à encoches.

Table 1: Inventory of normand sites having given notches adzes.

talon est la partie à laquelle on accordait le moins d'importance, la partie essentielle étant celle des encoches latérales liées à leur mode d'emmanchement.

Actuellement, sur le plan chronologique, le nombre de pièces attribuables à une période ou une culture néolithique donnée reste très indigent car les données sont souvent récoltées hors contexte archéologique fiable. On sait seulement que ces outils sont présents de la fin du Néolithique ancien jusqu'au Néolithique final, période à laquelle ils semblent beaucoup plus fréquents. On notera toutefois leur absence 

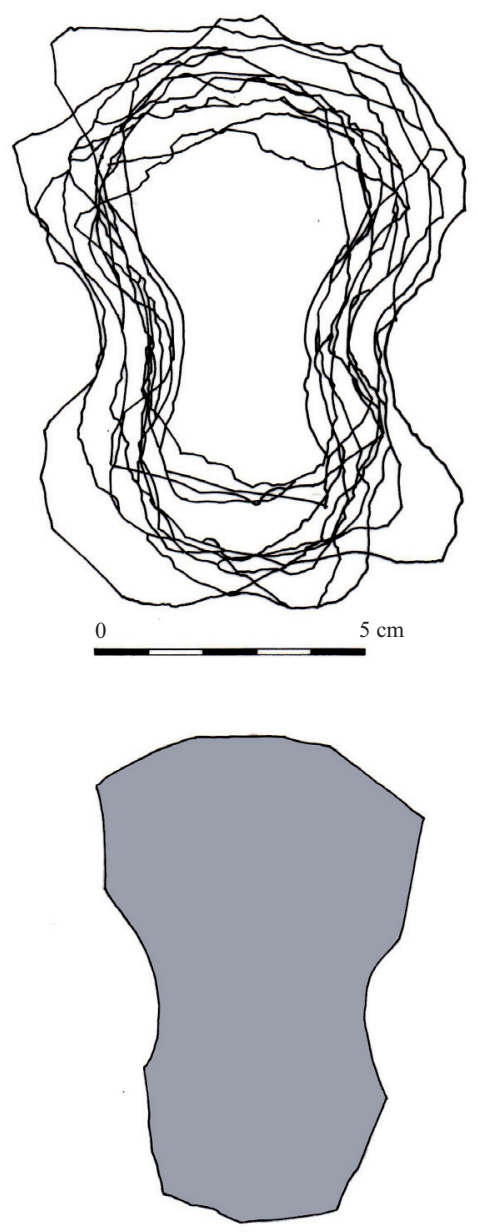

nettes à encoches, elle nous permet de proposer de tracer les premières limites de leur répartition géographique, limites qui, bien sûr, pourront être modifiées ultérieurement en fonction des découvertes à venir mais qui laissent l'espoir de définir dans le futur un territoire donné dans lequel ces outils particuliers ont été identifiés (fig. 16). Toutefois, la Normandie ne semble pas avoir l'exclusivité de ce type d'outils en France. D'autres régions plus ou moins éloignées pourraient y prétendre également. On se réfère au sud-ouest de la France où une herminette à encoches sur galet fut signalée sous le nom de " Hallebarde (lame de houe) » sur le site de "La Tuilerie " à Grépiac en Haute-Garonne, attribué au groupe de Véraza (Vaquer, 1980, p. 63, fig. 2).

\section{DisCUSSION CHRONOTYPOLOGIQUE ET GÉOTYPOLOGIQUE}

Tout d'abord, il convient de souligner que ces nouveaux outils que nous avons présentés en vue d'enrichir la liste typologique actuelle sur l'outillage néolithique qui a cours
Figure 14 : Portraitrobot des herminettes à encoches (dessin et DAO D. Prost).

Figure 14: Identikit picture of notched adzes. actuelle au Cerny et leur présence au BVSG final se limite au site de Bouafles "La Plante-à-Tabac " par une unique pièce (fig. 13, $\mathrm{n}^{0} 1$ ). La relative variabilité morphotypologique est visible mais ne peut être imputée à une variabilité chronologique. Il semble par contre beaucoup plus intéressant de voir que les herminettes à encoches sont limitées à un espace géographique restreint puisqu'on a l'habitude de ne les mentionner officiellement qu'en Normandie orientale (ancienne Haute-Normandie). Toutefois, en Normandie occidentale, Cayeux avait signalé la découverte ancienne d'une unique herminette à encoches à Clécy "Les Anneaux " dans le Calvados (Cayeux, 1957). Mais selon J.-P. Watté, il s'agirait d'un débitage naturel (Watté, 1998). Plus récemment, sur le site néolithique de Sevrai « La Haute-Rivière » fouillé par D. Giazzon dans l'Orne (Giazzon et al., 2014), nous avons pu identifier deux herminettes à encoches typiques dont l'une d'entre elles est très similaire au portrait-robot que nous en avons fait à partir des échantillons de Normandie orientale avant sa découverte (fig. 15), ce qui suggère une extension probable de ces outils en Normandie occidentale. Une étude cartographique des sites ayant fourni des hermi-

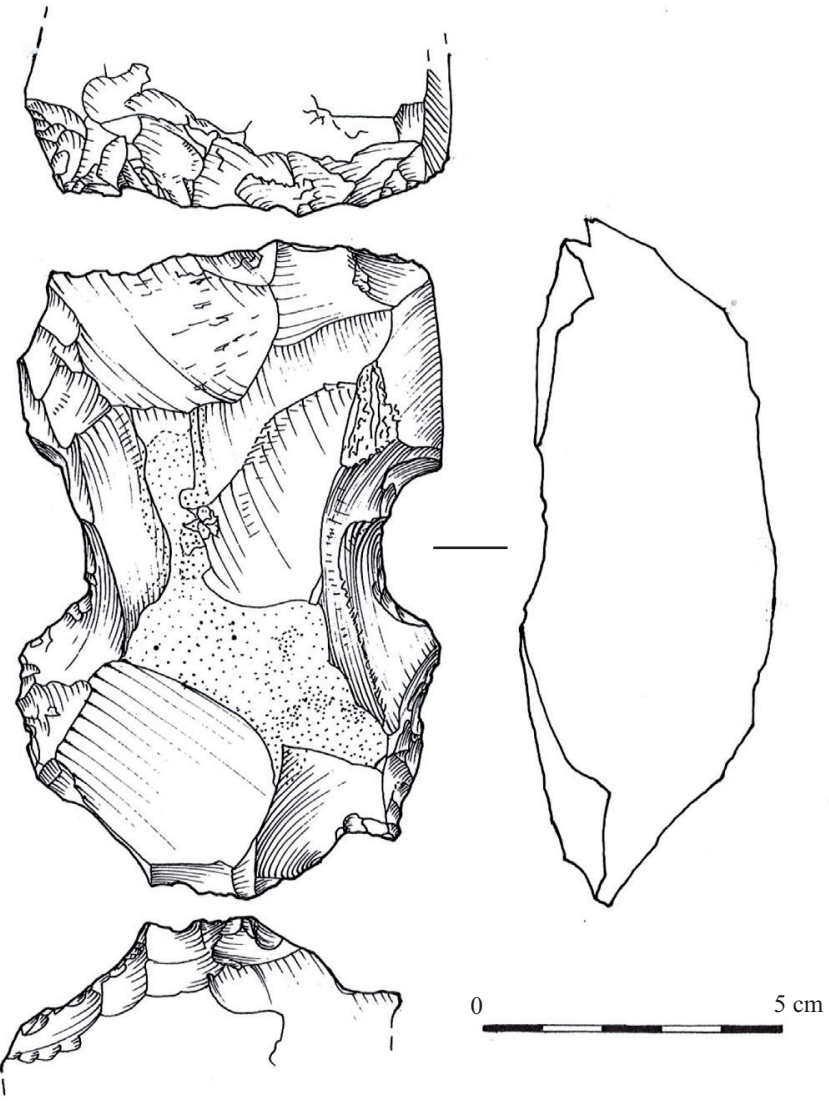

Figure 15 : Herminette à encoches de Sevray "La Haute-Rivière " (61) (dessin et DAO D. Prost).

Figure 15: Notched adzes from Sevray "La Haute-Rivière" (61). 


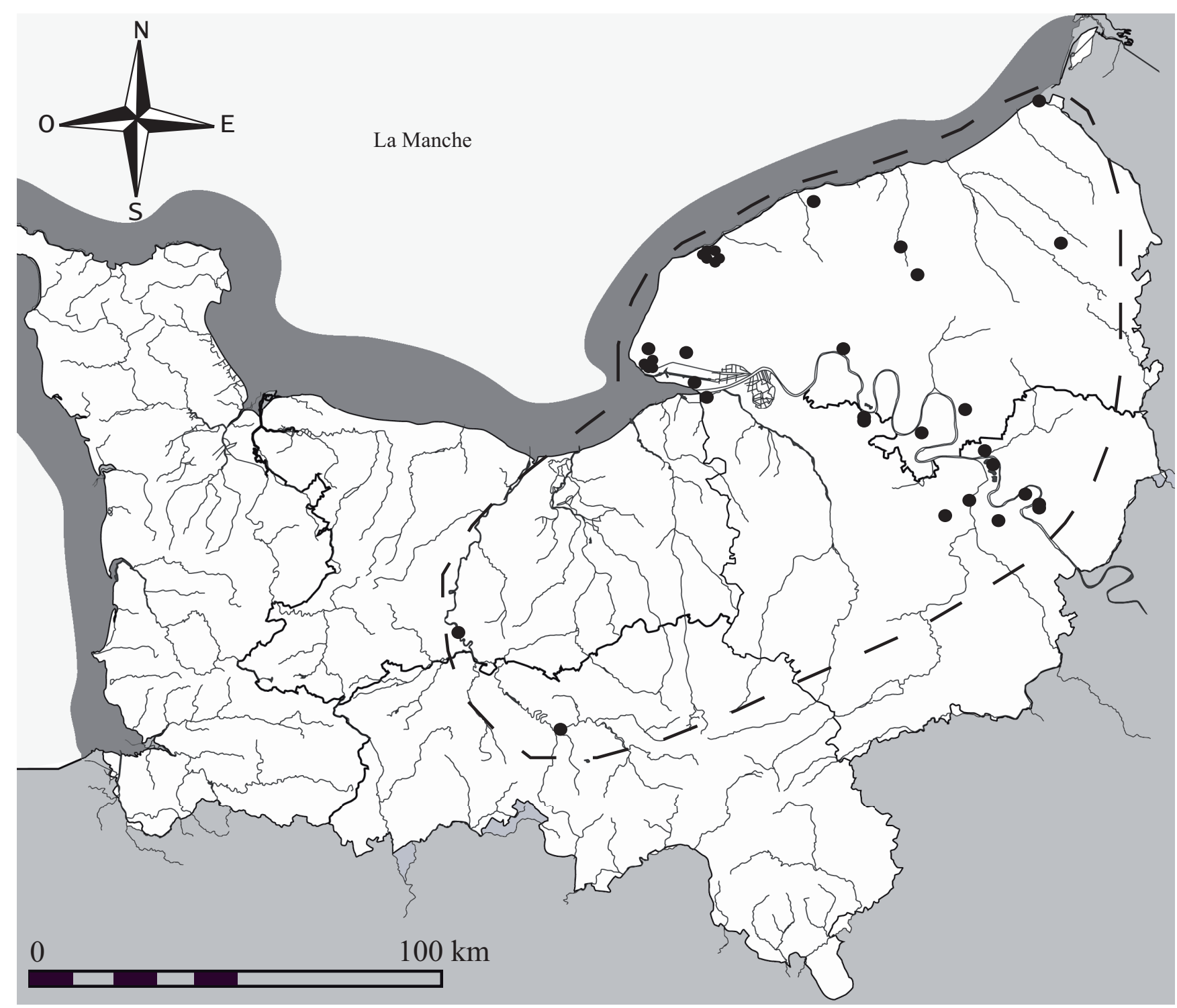

Figure 16 : Carte de répartition des sites producteurs d'herminettes à encoches en Normandie (DAO D. Prost).

Figure 16: Distribution map of sites that have produced notched adzes in Normandy.

actuellement dans le nord-ouest de la France restent une proposition soumise à discussion pour les reconnaître - ou non - comme des morphotypes à part. Si certains d'entre eux, comme les herminettes à encoches et, dans une moindre mesure, les couteaux quadrangulaires, semblent acceptés tout au moins par les néolithiciens normands -, il en va peut-être autrement pour les autres outils pour lesquels il faudra peut-être attendre d'autres découvertes par d'autres acteurs que l'auteur de cet article pour s'assurer - ou non - de leur reconnaissance typologique en Normandie mais aussi dans les régions limitrophes (Hauts-de-France, Île-deFrance, Centre-Val de Loire), voire au-delà.
Si on se réfere au tableau 2, chronologiquement, tous les outils que nous avons décrits s'illustrent par leur présence au Néolithique final. Deux d'entre eux (les becs biseautés et les pointes laminaires à coches basales) pourraient faire leur apparition au Chasséen septentrional. Il existe toutefois une césure entre le Néolithique moyen II et le Néolithique final, faute de séries lithiques du Néolithique récent bien conservées dans le Nord-Ouest qui pose la question d'une continuité d'existence de ces outils ou d'une phase d'interruption. Par ailleurs, la majorité de ces outils fut trouvée dans des sites localisés en Normandie orientale avec des extensions possibles dans la Somme, en Normandie occidentale dans le Calvados et l'Orne, voire en Île-de-France. Il conviendrait 


\begin{tabular}{|c|c|c|}
\hline Morphotypes & Périodes & Départements \\
\hline Becs biseautés & Chasséen et Néolithique récent-final & Eure, Seine-et-Marne, Somme \\
\hline Burins transversaux & Néolithique final - début Bronze ancien & Eure, Seine-Maritime, Somme \\
\hline Couteaux $1 / 2$ lune & Néolithique récent-final & Eure \\
\hline Couteaux quadrangulaires & Néolithique final & Eure, Seine-Maritime, Somme \\
\hline Pointes à coches basales & Chasséen et Néolithique récent-final & Eure, Seine-Maritime, Somme \\
\hline Herminettes à encoches & de la fin BVSG au Néolithique final & Calvados, Eure, Orne, Seine-Maritime \\
\hline
\end{tabular}

Tableau 2 : Inventaire chronologique et géographique par département des outils marginaux. Table 2: Chronological and geographical synthesis by departement of marginal tools.

donc à l'avenir de suivre leur extension chronologique et géographique et d'en définir peu à peu les limites.

Les herminettes à encoches nous offrent un premier exemple. Si elles sont susceptibles d'être présentes à plusieurs périodes du Néolithique, en revanche, le nombre de pièces trouvées autorise à proposer un premier tracé de leur extension géographique qui révèlerait un espace limité au sein de la Normandie (fig. 16). Ce type d'outils semble correspondre fidèlement à une des composantes matérielles qui pourrait servir de modèle culturel pour caractériser les cultures néolithiques que propose Alain Gallay : "Certaines particularités culturelles sont très étroitement localisées au plan géographique mais affichant des durées de vie exceptionnellement longues. Nous sommes ici en présence de traditions » (Gallay, 2016, p. 17). Aboutissant au fait que ces outils pouvaient avoir été emmanchés comme des lames d'herminettes, L. Cayeux entreprit de proposer une hypothèse fonctionnelle à ces outils. Il est amené à évoquer la fonction de « houe agricole plus adaptée aux travaux aratoires" (Cayeux, 1957, p. 574). Or, bien qu'aucune étude tracéologique n'ait encore été entreprise pour vérifier cette ancienne hypothèse, selon Leroi-Gourhan, la houe " porte très clairement l'empreinte du groupe qui l'utilise et que dans ce groupe elle garde avec fidélité sa forme, de génération en génération "(Leroi-Gourhan, 1973, p. 121). Il précise par la suite que la houe est fabriquée avec des moyens simples, économiques, aussi locaux que possible, traversant toute l'histoire d'un pays sans changer de forme, ce qui semble correspondre parfaitement à nos herminettes à encoches.

Quoi qu'il en soit, ces différents outils, que nous qualifions de marginaux, se distinguent par leur rareté, leur morphologie particulière, en marge du fonds commun de l'outillage néolithique. Ceci n'est pas nouveau, les néolithiciens ont repéré ce type d'outils depuis des décennies mais n'ont pas pris l'habitude d'y prêter beaucoup d'attention. Toutefois, les mentalités commencent à changer. Pour l'outillage chasséen par exemple, on reconnaît qu' « au-delà $\mathrm{du}$ fonds commun, de multiples différences se font jour au sein des mobiliers qui apparaissent de manière marginale "
(Augereau et al., 2016, p. 231). On commence à prendre conscience ici ou là de l'importance qu'il faudrait désormais accorder à ces outils marginaux dans l'étude de l'outillage lithique. Les outils communs auxquels on accorde l'essentiel de notre attention comme les grattoirs, les tranchets-ciseaux, les denticulés, les burins, les couteaux et les pièces à dos, les perçoirs, les pièces esquillées, les pièces à coches, etc., ont une diffusion chronologique et géographique souvent très large qui dépasse fréquemment la frontière culturelle qu'ils seraient censés caractériser. Dans le même état d'esprit, bien qu'en moindre quantité, on accorde aussi beaucoup d'importance à certains outils à cause de leur haute qualité technologique, la matière noble dont ils sont issus ainsi que la fonction ou la destination particulière qu'on leur prête comme les lames de haches polies et taillées, les poignards du Grand-Pressigny, les armatures de flèches dont la diffusion chronologique et/ou géographique est souvent très large également. Parmi ces outils, certains d'entre eux ont des origines très anciennes comme les grattoirs destinés au travail des peaux, identifiés depuis le Paléolithique supérieur (Gosselin, 2005) ou des origines liées au mode de vie sédentaire des premiers agriculteurs-éleveurs dont l'usage s'est répandu sur plusieurs continents (exemple des lames de haches polies). De ce fait, ces outils, en général, n’ont donc pas vocation en tant que morphotypes à servir de chronotypes ou de géotypes fiables pour définir une période ou une culture néolithique donnée. On tend à s'appuyer toutefois sur la variabilité de la fréquence de certains de ces outils, voire leur présence/absence, comme les microdenticulés, qui pourraient servir avec plus d'assurance d'indices chronoculturels. Reconnus comme marqueurs du Néolithique final, on sait aussi qu'ils perdurent au Bronze ancien et moyen. Leur fréquence pouvant différer d'un secteur géographique à l'autre, la discussion est ouverte pour savoir si cela peut contribuer à définir l'existence ou non de cultures ou de traditions distinctes. Mais quand on tente d'étudier ces outils de fonds commun de plus près, on est amené parfois à faire des distinctions d'ordre typologique et tracéologique, tradui- 
sant des fonctions ou des activités plus diversifiées qu'on ne l'imaginait. C'est le cas, par exemple dans le Nord-Ouest, des travaux sur les burins sur lame du BVSG (Philibert, 1996), sur certains grattoirs emmanchés comme herminettes (Caspar et Burnez-Lanotte, 1996; Gosselin in Prost et al., 2017) ou sur les tranchets chasséens de Bernières-sur-Seine (Prost et al., 2017). Les types d'outils qui se font moins fréquents, voire plus rares et, parmi eux, ceux destinés à être emmanchés, comme c'est le cas des racloirs à encoches, de certains couteaux ou pièces à moissonner, d'éléments de faucille, de certains grattoirs, etc., semblent plus favorables à servir de chronotypes. L'emmanchement peut être, dans ce cas, un facteur intéressant car le mode d'emmanchement exige de l'outil en silex qu'il ait une morphologie et des dimensions qui lui soient adaptées. De ce fait, la marque de fabrique de l'outil en silex, étroitement dépendante du manche, limite le choix du support, sa variabilité morphologique et typométrique car il doit répondre à des normes plus strictes que les outils pris en main. Ces exigences sont au premier plan d'ordre fonctionnel. Mais elles peuvent se traduire en termes de chronotypologie ou de géotypologie, par l'intermédiaire du type d'emmanchement si elles sont liées à une durée de vie courte (chronotype) et/ou une extension géographique restreinte (géotype). On est donc amené à formuler le fait que, si un outil a le profil d'être rare et emmanché, comme c'est le cas principalement des outils que nous présentons, on peut s'attendre de leur part d'avoir plus de chance d'être des chronotypes ou des géotypes que les autres. Ce serait là un sujet de discussion qui justifierait au moins qu'on s'attache de plus près à ces outils marginaux.

\section{Conclusion}

Au terme de cette étude qui présentait six outils pouvant contribuer à enrichir la typologie des outils néolithiques actuellement en vigueur dans le nord-ouest de la France, nous invitons les néolithiciens à vérifier leur validité morphotypologique sur d'autres sites. Ces outils pourraient servir d'exemples pour élargir la recherche sur les outils dits marginaux et discuter de la place qu'on pourrait leur accorder dans la détermination de l'outillage des groupes chronoculturels afin d'enrichir la liste restreinte des chronotypes (marquant leur appartenance à une période ou une culture donnée) et des géotypes (marquant leur appartenance à une aire géographique limitée) actuels. Le dernier colloque sur le Chasséen en 2014 fut une bonne illustration pour montrer que rien n'est figé en la matière. La notion de «Chasséen septentrional ", par exemple, qui affecte notre région, étant remise en question, les repères chronologiques sur lesquels nous avions l'habitude de nous baser devront être révisés et cela avec plus d'acuité à l'avenir (Perrin et al., 2016); ce qui justifie que l'on soit toujours à la recherche d'indices chronoculturels plus fréquents, plus fiables. L'outillage lithique, bien souvent écarté de cette discussion, pourrait y apporter une juste contribution en tenant compte de toutes ces composantes y compris celles marginales.

\section{Bibliographie}

Augereau A., Bostyn F., Garmond N., 2016 - « Impacts de la circulation des objets et des idées dans la structuration des assemblages lithiques chasséens dans le bassin de la Seine ", in Perrin T., Chambon P., Gibaja J.-F., Goude G. (dir), Le Chasséen, des Chasséens... Retour sur une culture nationale et ses parallèles, sépulcres de fossa, Cortaillod, Lagozza, actes du colloque international (Paris, 18-20 novembre 2014), Toulouse, Archives d'Écologie Préhistorique, p. 221-235.

BrÉzILlon M., 1971 - La dénomination des objets de pierre taillée, matériaux pour un vocabulaire des préhistoriens de langue française, Paris, Éditions du CNRS, 423 p. (Gallia Préhistoire, $4{ }^{\mathrm{e}}$ supplément).

Beurion C. (dir.), Deshayes T., Douillard A., LeclerHuby E., Le Maho S., Prost D., Santiago V., Tessier V., Thomann A., 2013 - Bardouville, Seine-Maritime "Le Moulin-à-Vent " (sablière Capoulade), rapport final d'opération, Cesson-Sévigné/Rouen, Inrap Grand-Ouest/SRA de HauteNormandie, 108 p.

Caspar J.-P., Burnez-Lanotte L., 1996 - « Groupe de BlicquyVilleneuve-Saint-Germain, nouveaux outils : le grattoir-herminette et le foret ", Bulletin de la Société préhistorique française, 93, 2, p. 235-240.

Cayeux L., 1957 - "Les herminettes et les houes à encoches latérales du Précampignien du Pays de Caux ", Bulletin de la Société préhistorique française, 54, 10, p. 572-575, 1 fig.

Cayeux L., 1970 - "La station précampignienne de l'ancien hameau de "La Mare Rouge" (Le Havre)", Bulletin de la Société d'Archéologie Préhistorique et Historique, p. 1-8.

Dubus A., 1910 - « Note sur la station préhistorique des Hogues près Yport ", Bulletin de la Société géologique de Normandie, 29 , p. $27-40$.

Duteurtre M., 1912 - « Les silex taillés de la forêt de Montgeon, le Val-Reinette", Bulletin de la Société géologique de Normandie, 31.

Fromont N. (dir.), Juhel L., NoëL J.-Y., Coutard S., Théron V., Beugnet L., Chantreuil L., Cottard A., Néré E., Biard M., Tessier V., Kerrouche V., 2006 Mesnil-Esnard "Les Hautes Haies ", rapport final d'opération, Cesson-Sévigné/Rouen, Inrap Grand-Ouest/SRA de HauteNormandie, $132 \mathrm{p}$.

Fromont N., Juhel L., Nö̈l J.-Y., Aubry B., 2013 - « MesnilEsnard "Les Hautes-Haies" (Seine-Maritime), une occupation 
du III millénaire av. J.-C. ", Revue archéologique de l'Ouest, 30, p. 57-86.

Gallay A., 2016 - "Quelques réflexions sur le concept de culture ", dans Perrin T., Chambon P., Gibaja J.-F., Goude G. (dir), Le Chasséen, des Chasséens... Retour sur une culture nationale et ses parallèles, sépulcres de fossa, Cortaillod, Lagozza, actes du colloque international (Paris, 18-20 novembre 2014), Toulouse, Archives d'Écologie Préhistorique, p. 11-23.

Giazzon D., Ghesquière E., Marcigny C., Prost D., 2014 Sevrai "La Haute-Rivière ", Orne, occupations du Néolithique ancien, du début du Néolithique récent et du Bronze ancien, rapport final d'opération, Cesson-Sévigné/Caen, Inrap GrandOuest/SRA de Basse-Normandie, 192 p.

Gosselin R., 2005 - "La transition Magdalénien-Azilien, observée à travers la fonction des grattoirs du Bois-Ragot ", dans Chollet A., Dujardin V. (dir.), La grotte du BoisRagot (Vienne) - Magdalénien et Azilien - Essai sur les hommes et leur environnement, Société préhistorique française (Mémoire XXXVIII), p. 191-219.

Honoré D. (dir.), 2007 - Honguemare-Guenouville, BourgHachard et Bosgouet "La Ferme-du-Pin ", "Le MoulinVacquet " et la ZAC du parc d'activité du Roumois, rapport final d'opération, Cesson-Sévigné/Rouen, Inrap Grand-Ouest/SRA de Haute-Normandie, 53 p.

Kliesch F. (dir.), 2012 - Le Tréport "Rue Dixon ", rapport final d'opération, Cesson-Sévigné/Rouen, Inrap Grand-Ouest/SRA de Haute-Normandie.

Lefèbvre G., Prost D., 2016 - « Prospection d'un site néolithique à Saint-Samson-de-la-Roqaue "Le Castel » (Eure, HauteNormandie), étude du mobilier lithique ", Haute-Normandie Archéologique, 18, Rouen, CRAHN-SNEP, p. 9-42.

Lepert T., 1987 - Saint-Wandrille-Rançon "Manoir-du-Perroy ", rapport de sauvetage programmé sur le site néolithique n 76-659002, SRA de Haute-Normandie, annexe 1 : inventaire des sites normands ayant livré des herminettes à encoches.

Leroi-Gourhan A., 1973 - Milieu et techniques, Paris, Albin Michel, 475 p. (Sciences d'aujourd'hui).

Marcigny C. (dir.) avec les contributions de Blanchet S., Boujot C., Carpentier V., Clément-Sauleau S., Corde D., Deloze V., Dietch-Sellami M.-F., Ghesquière E., Giazzon D., Gallouin E., Hugot C., Riche C., Leclerc E., Le Paumier H., Prost D., Sellami F., 2002 - Saint-VigorD’Ymonville (Seine-Maritime) "Les Sapinettes " et "La Mare des Mares " (Carrière Lafarge), document final de synthèse de fouille préventive, Cesson-Sévigné/Rouen, Inrap GrandOuest/SRA de Haute-Normandie, 4 vol.

Martin J.-M. (dir.), avec la participation de Marcon V., Martinez R., Prost D. et la collaboration de Bouillot J., Matterne V., Munod A.-V., Pernaud J.-M., 1996 Bettencourt-Saint-Ouen (n 80100003 AP) (Somme), Coordination A16, document final de synthèse de sauvetage urgent programmé, Afan avec le concours de la SANEF/SRA de Picardie, 2 vol.

Martinez R. et Prost D. (dir.), Biard M., Boulay G., Léon G., 1999 - Guerny (Eure) "Le Bois-Madame » déviation RN14, un site stratifié Campaniforme et Bronze ancien, document final de synthèse de fouille de sauvetage, Rouen, Afan/SRA de HauteNormandie.

Perrin T., Chambon P., Gibaja J.-F., Goude G. (dir), 2016 - Le Chasséen, des Chasséens... Retour sur une culture nationale et ses parallèles, sépulcres de fossa, Cortaillod, Lagozza, actes du colloque international (Paris, 18-20 novembre 2014), Toulouse, Archives d'Écologie Préhistorique, 556 p.

Philibert S., 1996 - « Approche techno-fonctionnelle des outillages du Néolithique ancien et moyen dans le Bassin parisien. Les gisements du Moulin-de-Lettrée (Neauphle-le-Vieux, Yvelines) et de Louviers "La Villette " (Eure) : premiers résultats ", Internéo 1, journée d'information du 23 novembre 1996, Paris, Saint-Germain en Laye/Paris, Association INTERNÉO/ Société préhistorique française, p. 33-44.

Prost D., 1992 - Le matériel lithique des prospections pédestres secteur IV du plateau de Marne-la-Vallée (Prospections G. Jacobiesski et al., 1986-1989) : inventaire, premières analyses, premiers résultats, rapport d'étude, Afan, Marne-la-Vallée Archéologie, base de Serris, SRA d'Île-de-France, $31 \mathrm{p}$.

Prost D., Biard M., 2000 - Bernières-sur-Seine "Les Fondriaux " (Eure), industrie lithique des niveaux chasséens (secteurs I, II et III), rapport d'étude, Afan/SRA de Haute-Normandie, carrière Lafarge-granulat.

Prost D., Boulay G., Théron V., 2004 - Bouafles (Eure), carrière R.D. $n^{\circ}$ 313, phase 1, rapport final d'opération de diagnostic, Cesson-Sévigné/Rouen/Paris, Inrap Grand-Ouest/SRA de Haute-Normandie/Morillon-Corvol, 8 p.

Prost D. (dir.), Boulay G., Forré P., Fromont N., Théron V., 2005 - Bouafles (27097), "La Plante-à-Tabac " parcelles 73 à 83, section D, carrière Morillon-Corvol, rapport final d'opération de fouille préventive, Cesson-Sévigné/Rouen/Paris, Inrap GrandOuest/SRA de Haute-Normandie/Morillon-Corvol, 61 p.

Prost D. (dir.), Langevin O., Lucquin A., March R., Verdin P., LÉPinay D., 2012 - Gaillon, Haute-Normandie, Eure (27) "La Garenne ", carrière Lafarge-Granulat: une occupation du Néolithique récent-final, rapport final d'opération de fouille préventive, Cesson-Sévigné/Rouen, Inrap GrandOuest/SRA de Haute-Normandie, 179 p.

Prost D., Langevin O., Lucquin A., March R.-J., Verdin P., LÉPINAY D., 2011 - "Le site Néolithique récent-final de "La Garenne" à Gaillon (Eure) et ses structures de combustion ", dans Bostyn F., Martial E., Praud I. (dir.), Le Néolithique $d u$ nord de la France dans son contexte européen, actes $d u$ $29^{\circ}$ colloque interrégional sur le Néolithique (Villeneuve-d'Ascq, 2-3 octobre 2009), Revue archéologique de Picardie, $n^{\circ}$ spécial, 28, p. 221-248. 
Prost D., Biard M., Gosselin R., Deloze V., Lépinay D., 2017 - «L'industrie lithique chasséenne de Bernières/Seine "Les Fondriaux" (Eure) », Gallia Préhistoire, 57, p. 337-372.

Riche C. (dir.), à paraître - Gaillon "La Garenne » (Eure), rapport final d'opération de fouille préventive, Cesson-Sévignél Rouen/Paris, Inrap Grand-Ouest/SRA de Haute-Normandie/ Lafarge-Granulat.

Thevenot J.-P., Strahm C. (dir.), 1976 - « La civilisation SaôneRhône ", Revue archéologique de l'Est et du Centre, 27, 3 et 4, p. 331-420.

Tixier J., Inizan M.-L., Roche H., 1980 - Préhistoire de la pierre taillée, 1 - Terminologie et technologie, Meudon, Cercle de recherche et d'études préhistoriques (CREP), $120 \mathrm{p}$.

VAQUer J., 1980 - « Le gisement de La Tuilerie de Grépiac (HauteGaronne) ", dans Guilaine J. (dir.), Le groupe de Véraza et la fin des temps néolithiques dans le sud de la France et la Catalogne, Paris, CNRS, p. 61-63.
VARIN W. (dir.), à paraître - Mesnil-Esnard, "route de Darnétal » (Seine-Maritime), rapport final d'opération de fouille préventive, Cesson-Sévigné/Rouen, Inrap Grand-Ouest/SRA de Haute-Normandie.

VignaRd E., 1929 - «Station aurignacienne du Champ de Bagasse à Mag-Hamadi (Haute-Égypte) ", Bulletin de la Société préhistorique française, 26, p. 299-306.

VignaRd E., 1934 - "Burin transversal et pseudo-microburin ", CPF, $11^{e}$ scession, Périgueux (Paris, 1935), p. 441-454.

Watté J.-P., 1991 - Le Néolithique en Seine-Maritime, Le Havre, éditions du Museum du Havre (supplément au Bulletin trimestriel de la Société Géologique de Normandie et des Amis du Museum du Havre, 77, 2).

Watté J.-P., 1998 - «Diagnostic archéologique sur le site Le Mont-Gaillard, rue de la Bigne-à-Fosse, Le Havre (SeineMaritime) ", Annales du Museum du Havre, 58, p. 3-15. 